\title{
A complete description of the dynamics of legal outer-totalistic affine continuous cellular automata
}

\section{Barbara Wolnik}

Gdansk University: Uniwersytet Gdanski

Marcin Dembowski ( $\nabla$ marcin.dembowski@gmail.com )

Gdansk University: Uniwersytet Gdanski https://orcid.org/0000-0002-5824-9779

Antonii Augustynowicz

Uniwersytet Gdański: Uniwersytet Gdanski

\section{Bernard De Baets}

Ghent University: Universiteit Gent

\section{Research Article}

Keywords: Affine Continuous Cellular Automata, Outer Totalistic Cellular Automata

Posted Date: April 6th, 2022

DOI: https://doi.org/10.21203/rs.3.rs-745409/v2

License: (c) (i) This work is licensed under a Creative Commons Attribution 4.0 International License. Read Full License 


\title{
A complete description of the dynamics of legal outer-totalistic affine continuous cellular automata
}

\author{
Barbara Wolnik @ Marcin Dembowski @ Antoni Augustynowicz $@$ \\ Bernard De Baets
}

Received: date / Accepted: date

\begin{abstract}
This paper presents an investigation into the evolution and dynamics of the simplest generalization of binary cellular automata: Affine Continuous Cellular Automata (ACCAs), with $[0,1]$ as state set and local rules that are affine in each variable. The focus lies on legal outer-totalistic ACCAs, an interesting class of dynamical systems that show some properties that do not occur in the binary case. A unique combination of computer simulations (sometimes quite advanced) and a panoply of analytical methods allow to lay bare the dynamics of each and every one of these cellular automata. The results show that in the class of ACCAs considered, one can observe all types of sensitivity: sensitivity to the change of the number of cells in the grid, sensitivity to slight changes in the parameters of a local rule and sensitivity to the change of a single value in an initial configuration.
\end{abstract}

Keywords Affine Continuous Cellular Automata . Outer Totalistic Cellular Automata

Mathematics Subject Classification (2020) MSC 37B15, MSC 68Q80

Barbara Wolnik

Institute of Mathematics, Faculty of Mathematics, Physics and Informatics, University of Gdańsk, 80-308 Gdańsk, Poland

Marcin Dembowski

Gdańsk, Poland E-mail: marcin.dembowski@gmail.com

Antoni Augustynowicz

Institute of Mathematics, Faculty of Mathematics, Physics and Informatics, University of Gdańsk, 80-308 Gdańsk, Poland

Bernard De Baets

KERMIT, Department of Data Analysis and Mathematical Modelling, Ghent University, 9000 Gent, Belgium

\section{Introduction}

Continuous Cellular Automata (CCAs) can be seen as a generalization of Cellular Automata (CAs), in which time and space are still discrete, but cells can take states from some infinite (often continuous) set. One of the best known examples of such dynamical systems are coupled map lattices [1,2,3. An exploration of the dynamics of CCAs is very difficult and is often performed through computer simulation only (see, for example, [3, 4, 5, 6, 7]). Not surprisingly then, because of their dependence on computer precision, there is limited confidence in the results obtained. Also, it is not obvious how convergence should be decided upon. Moreover, in computer simulations, one usually sets a certain ("large enough") number of cells, a practice that is not favorable for at least two reasons. First, it is impossible to test all initial configurations, even if one is only interested in the binary ones. Second, oftentimes CAs behave differently depending on the number of cells (for example, the dependence on parity in the case of CAs with radius 1). Moreover, when the phenomena observed, such as bifurcations or phase transitions, are characterized by numerical parameters, the values of the latter can only be found approximately. However, some studies complement an experimental study by insightful analytical research that confirms and further refined the results (see, for example, 8, 8, 10]).

A well-known generalization of binary CAs to CCAs with $[0,1]$ as state set is obtained by "fuzzification" in 4 and was further studied, for example, in 8, 11. This fuzzification process allows to associate with every binary CA some particular CCA (a so-called Fuzzy $\mathrm{CA}$ ) through an extension of the domain of the local rule. The relationship between Fuzzy CAs and Elementary CAs (ECAs) can be found in 12. The dynamics 
of some FCAs (called weighted average rules) is theoretically explained in [8]. The detailed analytical study presented in said paper confirmed, for example, the empirical observation that all weighted average rules are periodic in time and space.

A further generalization of the above idea has resulted in the definition of affine CCAs (ACCAs) [13]. This kind of CCAs is considered the simplest possible generalization of binary CAs - of course, apart from the Fuzzy CAs - as they have a local rule that is affine in each variable. It appears that ACCAs offer much richer behavior than the binary CAs they stem from. For example, in 9] density-conserving ACCAs are considered and it is shown that most of them can solve the relaxed density classification problem, which is not possible with binary CAs. This result was obtained through a theoretical study of the dynamics of density-conserving ACCAs.

In this paper, we focus on one-dimensional ACCAs (with radius 1 ) that are both outer-totalistic and legal. The first property means that such ACCAs update the value of a cell only on the basis of the current value of the cell and the sum of values of all other cells in the neighborhood of that cell. The second property ensures that nothing can be produced from zeros. A combination of these two properties is very often used when modeling various phenomena. For example, it is a basic assumption in each "full of common sense" Life-like CA, i.e., being similar to Conway's Game of Life 14, 15. Our motivation to consider one-dimensional ACCAs only is rather simple: we have set ourselves the goal to explore the dynamics theoretically and not by analyzing computer simulations only. As will become clear in this paper, even in the one-dimensional case this is a challenging task. Up to now, there are no such theoretical results on the dynamics of two-dimensional cellular automata, such as the mentioned Game of Life, despite the ample interest of researchers (see, for instance, 16. 17, 18). Moreover, even in the case of one-dimensional cellular automata, virtually all existing methods describe the dynamics on the basis of computer simulations (see, for instance, [19,20]).

Among the ECAs there are 64 outer-totalistic CAs. Only four of them are still outer-totalistic after the "fuzzification" process and show a very simple dynamics. Restricting our attention to the legal ones, we are left with only two ECAs, i.e., only two ECAs are still legal and outer-totalistic after fuzzification: ECA 0 (the zero rule) and ECA 204 (the identity rule). The situation looks quite different for ACCAs. There are infinitely many legal outer-totalistic ACCAs and their dynamics are very diverse, which will be amply demonstrated in this paper. To be more precise, we will un- cover the dynamics of all such ACCAs. Preliminary hypotheses will be generated on the basis of simulation results, but will then be confirmed analytically. More specifically, we will use the results of preliminary computer simulations (sometimes quite advanced) to identify the proper mathematical tools for our theoretical analysis. This approach will allow to lay bare the dynamics of each and every outer-totalistic legal ACCA. The results will show that in this class of cellular automata one can observe all types of sensitivity: sensitivity to the change of the number of cells in the grid, sensitivity to slight changes in the parameters of a local rule and sensitivity to the change of a single value in an initial configuration. It is safe to say that all the results presented in this paper are new (except when the rule under consideration is number-conserving, as this case was considered in [9]).

This paper is organized as follows. Definitions and theoretical facts are given in Section 2. Computational experiments are described in Section 3 , while the ACCA dynamics are discussed in Section 4 . The paper is concluded with Section 5 .

\section{Outer-totalistic ACCAs}

A CCA is a dynamical system consisting of a grid of cells, where each cell is assigned one of infinitely many states. We consider the one-dimensional case, radius 1 and periodic boundary conditions, so that the cells are arranged in a circular linear array and each cell has two adjacent cells, which together with the cell itself constitute its neighborhood.

As state set we consider the interval $[0,1]$, so that the local rule $f$ is a function from $[0,1]^{3}$ to $[0,1]$ and if the number of cells $N$ is given, it generates the global rule $F:[0,1]^{N} \rightarrow[0,1]^{N}$ in the usual way, i.e., for each $\boldsymbol{x} \in[0,1]^{N}$, we have

$F(\boldsymbol{x})_{n}=f\left(x_{n-1}, x_{n}, x_{n+1}\right)$,

where $n \in\{0,1, \ldots, N-1\}$ is the index of the $n$-th cell of the grid (all operations on the indices are performed modulo $N)$. Further, $F^{t}(\boldsymbol{x})$ denotes the result of the $t$-th application of $F$ to the configuration $\boldsymbol{x}$, corresponding to the $t$-th time step. Moreover, we will write $x_{n}^{t}$ to denote the value of the $n$-th cell in $F^{t}(\boldsymbol{x})$.

For a given configuration $\boldsymbol{x} \in[0,1]^{N}$, we define the sum of its states and its density respectively by

$\sigma(\boldsymbol{x})=\sum_{i=0}^{N-1} x_{i} \quad$ and $\quad \rho(\boldsymbol{x})=\frac{1}{N} \sigma(\boldsymbol{x})$.

Furthermore, for $\boldsymbol{x} \in[0,1]^{N}$ and $t \in \mathbb{N}$, we denote the minimal and maximal value among $x_{0}^{t}, x_{1}^{t}, \ldots, x_{N-1}^{t}$ 
by $\min \left(F^{t}(\boldsymbol{x})\right)$ and $\max \left(F^{t}(\boldsymbol{x})\right)$, respectively. The Euclidean norm in $\mathbb{R}^{N}$ is denoted by the symbol $\|\cdot\|$. For the sake of brevity, we often express configurations in a more compact form and write, for instance, $1^{2} 0^{4} 1(01)^{2}$ as a self-explanatory shorthand for 11000010101.

In this paper, we restrict our attention to the interesting class of affine CCAs (ACCAs), i.e., CCAs whose local rule is affine in each variable (see [9]). To define a function $f:[0,1]^{3} \rightarrow[0,1]$ that is affine in each variable, we start by defining the values of $f$ on $\{0,1\}^{3}$, i.e., we fix the values $l_{0}=f(0,0,0), l_{1}=f(0,0,1)$, $l_{2}=f(0,1,0), l_{3}=f(0,1,1), l_{4}=f(1,0,0), l_{5}=$ $f(1,0,1), l_{6}=f(1,1,0)$ and $l_{7}=f(1,1,1)$. The sequence $\left(l_{0}, l_{1}, \ldots, l_{7}\right) \in[0,1]^{8}$ is called the lookup table (LUT) of $f$. The general form of a LUT is given in Table 1. Next, we extend the function to the entire do-

Table 1: The general form of the LUT of the local rule of an ACCA.

\begin{tabular}{|c|c|c|c|c|c|c|c|}
\hline$(0,0,0)$ & $(0,0,1)$ & $(0,1,0)$ & $(0,1,1)$ & $(1,0,0)$ & $(1,0,1)$ & $(1,1,0)$ & $(1,1,1)$ \\
\hline$l_{0}$ & $l_{1}$ & $l_{2}$ & $l_{3}$ & $l_{4}$ & $l_{5}$ & $l_{6}$ & $l_{7}$ \\
\hline
\end{tabular}

main $[0,1]^{3}$. Obviously, the extension that is affine in each variable is unique and can be expressed as the following polynomial:

$$
\begin{aligned}
f(x, y, z)= & l_{0}(1-x)(1-y)(1-z) \\
& +l_{1}(1-x)(1-y) z \\
& +l_{2}(1-x) y(1-z)+l_{3}(1-x) y z \\
& +l_{4} x(1-y)(1-z)+l_{5} x(1-y) z \\
& +l_{6} x y(1-z)+l_{7} x y z .
\end{aligned}
$$

ACCAs are a generalization of Fuzzy CAs that are constructed from ECAs by "fuzzification" of their disjunctive normal form [21]. Thus, there are exactly 256 Fuzzy CAs, because each ECA corresponds to a unique Fuzzy CA, whose local rule is also defined by Eq. (1), where $l_{0}, \ldots, l_{7}$ are the LUT entries of the given ECA.

To understand the difference between ECAs, Fuzzy CAs and ACCAs, it is worth emphasizing that the local rules of all these CA families are defined by Eq. (1), but only in the case of ECAs both $\left(l_{0}, l_{1}, \ldots, l_{7}\right) \in\{0,1\}^{8}$ and $(x, y, z) \in\{0,1\}^{3}$. In the case of Fuzzy CAs, still $\left(l_{0}, l_{1}, \ldots, l_{7}\right) \in\{0,1\}^{8}$ but $(x, y, z) \in[0,1]^{3}$, while in the case of ACCAs both $\left(l_{0}, l_{1}, \ldots, l_{7}\right) \in[0,1]^{8}$ and $(x, y, z) \in[0,1]^{3}$.

In the investigations presented in this paper, we will further restrict our attention to legal outer-totalistic ACCAs. An outer-totalistic CA is a CA whose local rule depends only on the state of the focal cell and the sum of the states of the adjacent cells. The following theorem provides a necessary and sufficient condition for an ACCA to be outer-totalistic.

Theorem 1 A one-dimensional ACCA is outer-totalistic if and only if the entries of the LUT of its local rule satisfy

$$
\begin{cases}l_{0}=a & l_{4}=b \\ l_{1}=b & l_{5}=2 b-a \\ l_{2}=c & l_{6}=d \\ l_{3}=d & l_{7}=2 d-c\end{cases}
$$

where $a, b, c, d \in[0,1], \frac{1}{2} a \leq b \leq \frac{1}{2} a+\frac{1}{2}$ and $\frac{1}{2} c \leq d \leq$ $\frac{1}{2} c+\frac{1}{2}$. In other words, the local rule $f$ of an outertotalistic ACCA is given by the following formula

$$
\begin{aligned}
f(x, y, z)= & (d-c-b+a)(x+z) y \\
& +(b-a)(x+z)+(c-a) y+a .
\end{aligned}
$$

Proof It is easy to see that if $f$ is given by Eq. (3), with the restrictions that $a, b, c, d \in[0,1], \frac{1}{2} a \leq b \leq \frac{1}{2} a+\frac{1}{2}$ and $\frac{1}{2} c \leq d \leq \frac{1}{2} c+\frac{1}{2}$, the corresponding ACCA is outertotalistic. To prove that the conditions in Eq. (2) are necessary, it suffices to note that the following equalities have to hold:

$$
\left\{\begin{array}{l}
f(1,0,0)=f(0.5,0,0.5)=f(0,0,1) \\
f(1,1,0)=f(0.5,1,0.5)=f(0,1,1)
\end{array} .\right.
$$

Hence, from Eq. (1) we get

$$
\left\{\begin{array}{l}
l_{4}=\frac{1}{4}\left(l_{0}+l_{1}+l_{4}+l_{5}\right)=l_{1} \\
l_{6}=\frac{1}{4}\left(l_{2}+l_{3}+l_{6}+l_{7}\right)=l_{3}
\end{array},\right.
$$

which yields Eq. (2). Substituting Eq. (2) into Eq. (1), we then get Eq. (3).

Let us note that for Fuzzy CAs the necessary and sufficient condition for being outer-totalistic is exactly the same as for ACCAs in Theorem 1 with the additional assumption that $a, b, c, d \in\{0,1\}$. As a consequence, we obtain that among the 64 outer-totalistic ECAs, only four of them are still outer-totalistic when we extend the domain of their local rule from $\{0,1\}^{3}$ to $[0,1]^{3}$. These are: ECA 0, ECA 51 (the negation), ECA 204 (the identity) and ECA 255. Thus, there are only four outer-totalistic Fuzzy CAs with the following local rules: $f_{1}(x, y, z)=0, f_{2}(x, y, z)=1-y$, $f_{3}(x, y, z)=y$ and $f_{4}(x, y, z)=1$. All of these rules give rise to a very simple dynamics.

According to Theorem 1, the set of all outer-totalistic ACCAs is parameterized by points of the four-dimensional polytope that can be obtained as the Cartesian product $D \times D$, where the parallelogram $D$ has vertices $(0,0),(1,0.5),(1,1)$ and $(0,0.5)$. 
It is easy to see that if some ACCA is outer-totalistic and its local rule $f$ is parameterized by a point $(a, b, c, d)$, then the conjugate $\mathrm{CA}$, whose local rule $f^{C}$ is given by $f^{C}(x, y, z)=1-f(1-x, 1-y, 1-z)$, is also an outertotalistic ACCA. Moreover, $f^{C}$ is parameterized by the point $\left(a^{\prime}, b^{\prime}, c^{\prime}, d^{\prime}\right)$, where $a^{\prime}=1+c-2 d, b^{\prime}=1-d$, $c^{\prime}=1+a-2 b$ and $d^{\prime}=1-b$. Note that the behavior of $F^{C}$ is strongly related to the behavior of $F$. Indeed, if $\boldsymbol{x}$ is any configuration from $[0,1]^{N}$ and $\boldsymbol{y}$ is the configuration given by $y_{i}=1-x_{i}$, for $i \in\{0,1, \ldots, N-1\}$, then at each time step $t \geq 0$ it holds that $y_{i}^{t}=1-x_{i}^{t}$. For binary CAs the conjugation $F \mapsto F^{C}$ is often called the white-to-black transformation.

The following theorem shows a basic property of the local rule of ACCAs.

Theorem 2 Let $0 \leq m \leq M \leq 1$. If a function $f:[0,1]^{3} \rightarrow[0,1]$ is affine in each variable, then for any $x, y, z \in[m, M]$, it holds that

$\min (\mathcal{V}) \leq f(x, y, z) \leq \max (\mathcal{V})$

where the set $\mathcal{V}$ is defined as

$\mathcal{V}=\{f(x, y, z) \mid x, y, z \in\{m, M\}\}$.

We omit the proof, as it is an immediate consequence of the monotonicity of $f$ w.r.t. each variable.

In this paper, we focus on a subclass of outer-totalistic ACCAs, known as legal ACCAs: a CA is called legal if it cannot produce anything from zeros, i.e., its local rule $f$ satisfies $l_{0}=f(0,0,0)=0$. According to Eqs. (2) and (3), the local rule of a legal outer-totalistic ACCA is given by

$f(x, y, z)=(d-c-b)(x+z) y+b(x+z)+c y$,

where $0 \leq b \leq \frac{1}{2}, 0 \leq c \leq 1$ and $c \leq 2 d \leq c+1$. These restrictions on the parameters $b, c, d$ generate a parallelepiped $\mathcal{P}$ in the Cartesian coordinate system, which is shown in Figure 1 .

As one can see, the parallelepiped $\mathcal{P}$ has eight vertices, thus there are exactly eight extreme points in the set of all legal outer-totalistic ACCAs (see Table 2). The local rule of any legal outer-totalistic ACCA can be written as a convex combination of the local rules listed in the last column of Table 2 .

\section{Computer simulations}

Our goal is to examine the dynamics of all legal outertotalistic ACCAs, more specifically, we want to determine how these dynamics evolve when starting from binary configurations. We concentrate on such initial configurations for two reasons. Firstly, many problems

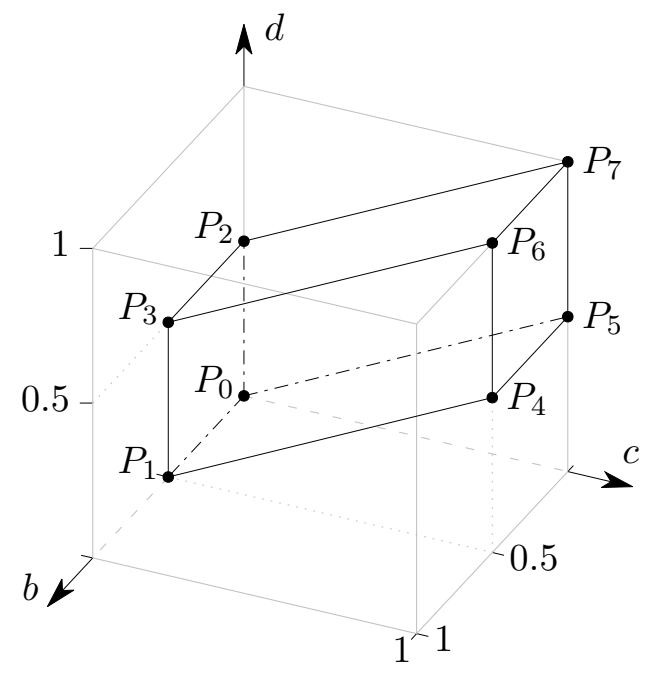

Fig. 1: Parameterization of the set of legal outertotalistic ACCAs.

Table 2: Analytical expressions of the local rules corresponding to the extreme points in the set of legal outer-totalistic ACCAs.

\begin{tabular}{|c|l|}
\hline Vertex & Corresponding local rule \\
\hline$P_{0}=(0,0,0)$ & $f(x, y, z)=0($ ECA 0$)$ \\
\hline$P_{1}=\left(\frac{1}{2}, 0,0\right)$ & $f(x, y, z)=\frac{1}{2}(x+z)(1-y)$ \\
\hline$P_{2}=\left(0,0, \frac{1}{2}\right)$ & $f(x, y, z)=\frac{1}{2}(x+z) y$ \\
\hline$P_{3}=\left(\frac{1}{2}, 0, \frac{1}{2}\right)$ & $f(x, y, z)=\frac{1}{2}(x+z)$ \\
\hline$P_{4}=\left(\frac{1}{2}, 1, \frac{1}{2}\right)$ & $f(x, y, z)=y+(x+z)\left(\frac{1}{2}-y\right)$ \\
\hline$P_{5}=\left(0,1, \frac{1}{2}\right)$ & $f(x, y, z)=y-\frac{1}{2}(x+z) y$ \\
\hline$P_{6}=\left(\frac{1}{2}, 1,1\right)$ & $f(x, y, z)=y+\frac{1}{2}(x+z)(1-y)$ \\
\hline$P_{7}=(0,1,1)$ & $f(x, y, z)=y($ ECA 204$)$ \\
\hline
\end{tabular}

concerning cellular automata are centered on binary configurations (as, for example, the density classification problem). Secondly, we want to deal with a finite set of initial configurations to be able to examine all of them using computer simulations.

Our investigation started with very detailed simulations, described below, whose results facilitated to formulate preliminary hypotheses (depending on the range of the parameters $b, c$ and $d$ ), some of which could be easily confirmed theoretically, while other ones required often very sophisticated additional simulations, revealing the underlying mechanism of a given ACCA in detail. This was essential in order to be able to choose the proper analytical tools and mathematical proof methods. As a result, it became possible to describe and theoretically confirm the dynamics of all legal outertotalistic ACCAs. Below, we present the experimental set-up for the first part of the simulations. 
If the number of cells $N$ is given, then $X_{N}$ denotes the set of all binary configurations of length $N$, i.e., $X_{N}=\{0,1\}^{N}$. Additionally, we use the notations $X_{N}^{0}$, $X_{N}^{1}$ and $X_{N}^{0,1}$ to refer to the set $X_{N}$ without $0^{N}, 1^{N}$ or both of these elements, respectively, i.e.,

$$
\begin{aligned}
X_{N}^{0} & =X_{N} \backslash\left\{0^{N}\right\}, X_{N}^{1}=X_{N} \backslash\left\{1^{N}\right\}, \\
X_{N}^{0,1} & =X_{N} \backslash\left\{0^{N}, 1^{N}\right\},
\end{aligned}
$$

where, as mentioned earlier, $0^{N}$ and $1^{N}$ are shorthand for $\underbrace{0 \ldots 0}_{N}$ and $\underbrace{1 \ldots 1}_{N}$.

In the first part of our experiments, we sampled the set of all legal outer-totalistic ACCAs parameterized by $(b, c, d)$ by varying the parameter $c$ from 0 to 1 with a step size of 0.1 and - taking into account Theorem 1 - the parameters $b, d$ from 0 to 0.5 and from $0.5 c$ to $0.5 c+0.5$, respectively, with a step size of 0.05 . For each such set of parameters, we defined the local rule $f$ by the expression in Eq. (4), resulting in a set $\mathcal{R}$ containing 1331 rules.

Because we did not want to be dependent on randomly selected configurations, we chose small $N$ and examined all binary configurations of this length. As our investigations conducted in a previous work [9] showed that the behavior of an ACCA may depend on whether $N$ is odd or even, we considered two specific sizes of the grid: $N=10$ and $N=11$. Note that $\left|X_{10}\right|=2^{10}$ and $\left|X_{11}\right|=2^{11}$. For every local rule $f \in \mathcal{R}$, we carried out the following simulations. For each $\boldsymbol{x} \in X_{N}$, we ran the corresponding global rule $F$ for $\Lambda=10000$ iterations and classified $\boldsymbol{x}$, by checking the output configurations $F^{\Lambda}(\boldsymbol{x}), F^{\Lambda-1}(\boldsymbol{x}), F^{\Lambda-2}(\boldsymbol{x})$, to the first group $G_{i}^{(N)}$ with a matching condition:

$G_{0}^{(N)}$ : all cell states are close to 0 and configurations $F^{\Lambda}(\boldsymbol{x})$ and $F^{\Lambda-1}(\boldsymbol{x})$ are almost the same, i.e., $\max \left(F^{\Lambda}(\boldsymbol{x})\right)<\varepsilon$ and $\left\|F^{\Lambda}(\boldsymbol{x})-F^{\Lambda-1}(\boldsymbol{x})\right\|<\eta$. In this case, we conjecture that $F^{t}(\boldsymbol{x})$ tends to $0^{N}$.

$G_{1}^{(N)}$ : all cell states are close to 1 and configurations $F^{\Lambda}(\boldsymbol{x})$ and $F^{\Lambda-1}(\boldsymbol{x})$ are almost the same, i.e., $\min \left(F^{\Lambda}(\boldsymbol{x})\right)>1-\varepsilon$ and $\left\|F^{\Lambda}(\boldsymbol{x})-F^{\Lambda-1}(\boldsymbol{x})\right\|<\eta$. In this case, we conjecture that $F^{t}(\boldsymbol{x})$ tends to $1^{N}$. $G_{2}^{(N)}$ : all cell states are close to the same value and configurations $F^{\Lambda}(\boldsymbol{x})$ and $F^{\Lambda-1}(\boldsymbol{x})$ are almost the same, i.e., $\max \left(F^{\Lambda}(\boldsymbol{x})\right)-\min \left(F^{\Lambda}(\boldsymbol{x})\right)<\varepsilon$ and $\left\|F^{\Lambda}(\boldsymbol{x})-F^{\Lambda-1}(\boldsymbol{x})\right\|<\eta$. In this case, we conjecture that $F^{t}(\boldsymbol{x})$ tends to $c^{N}$, for some $c \in(0,1)$ (the constant $c$ may depend on $\boldsymbol{x}$ ).

$G_{3}^{(N)}$ : all cell states are close to the same value and configurations $F^{\Lambda}(\boldsymbol{x})$ and $F^{\Lambda-2}(\boldsymbol{x})$ are almost the same, i.e., $\max \left(F^{\Lambda}(\boldsymbol{x})\right)-\min \left(F^{\Lambda}(\boldsymbol{x})\right)<\varepsilon$ and $\left\|F^{\Lambda}(\boldsymbol{x})-F^{\Lambda-2}(\boldsymbol{x})\right\|<\eta$. In this case, we conjecture that $F^{2 t}(\boldsymbol{x})$ tends to $c_{1}^{N}$ and $F^{2 t+1}(\boldsymbol{x})$ tends to $c_{2}^{N}$, for some $c_{1}, c_{2} \in[0,1]$ (a convergence with period 2).

$G_{4}^{(N)}$ : configurations $F^{\Lambda}(\boldsymbol{x})$ and $F^{\Lambda-1}(\boldsymbol{x})$ are almost the same, i.e., $\left\|F^{\Lambda}(\boldsymbol{x})-F^{\Lambda-1}(\boldsymbol{x})\right\|<\eta$. In this case, we assume that $F^{t}(\boldsymbol{x})$ tends to some fixed configuration.

$G_{5}^{(N)}$ : all other configurations.

We will drop the upper index $(N)$ unless confusion is possible.

For the reported experiments, we chose $\varepsilon=0.001$ and $\eta=0.01$. As a result, for every local rule $f \in$ $\mathcal{R}$, we obtained 12 subsets of binary configurations: $G_{0}^{(10)}, \ldots, G_{5}^{(10)}$ and $G_{0}^{(11)}, \ldots, G_{5}^{(11)}$, satisfying $G_{0}^{(10)} \cup$ $G_{1}^{(10)} \cup \ldots \cup G_{5}^{(10)}=X_{10}$ and $G_{0}^{(11)} \cup G_{1}^{(11)} \cup \ldots \cup G_{5}^{(11)}=$ $X_{11}$. On this basis, we formulated the preliminary hypothesis on the dynamics of $f$.

For example, for the local rule $f(x, y, z)=0.1(x+$ $z) y+0.5 y$, parameterized by the point $(b, c, d)=(0,0.5$, $0.6)$, the simulations yield $G_{0}^{(10)}=X_{10}$ and $G_{0}^{(11)}=$ $X_{11}$, (while the other subsets are empty). Thus, it is obvious that we conjecture that for each binary configuration $\boldsymbol{x} \in X_{N}$, it holds that $F^{t}(\boldsymbol{x})$ tends to $0^{N}$.

As mentioned above, the results of these first experiments allowed to detect the nature of many legal outer-totalistic ACCAs. Moreover, it is not hard to confirm these observations theoretically (see, for example, Subsection 4.3. However, for some local rules we were forced to perform additional simulations. In particular, we varied $N, \varepsilon, \eta$, and used various data exploration techniques that allow for tracking both the positions and values of $\min \left(F^{t}(\boldsymbol{x})\right)$ and $\max \left(F^{t}(\boldsymbol{x})\right)$. In some cases, we relied on a visualization of the dynamics.

To visualize the dynamics of an ACCA, we use a slight modification of the radial view of CCAs - a method proposed in 22. If the number of cells $N$ is given, we consider a circle with radius 1.1 and center $C$ divided in $N$ equal sectors by points $A_{0}, A_{1}, \ldots, A_{N-1}$. The value $x_{n}$ of the $n$-th cell is represented by a dot lying on the line segment $C A_{n}$ at a distance $0.1+x_{n}$ from $C$. For the sake of convenience, we connect subsequent dots using a polyline. Four examples of the radial view of some configurations from $[0,1]^{10}$ are shown in Figure 2.

The radial representations at subsequent time steps allow to observe the evolution of a given CCA and gain some intuition about the rules that govern it. For example, Figure 3 shows the dynamics of the ACCA with the local rule $f(\boldsymbol{x})=-0.25(x+z) y+0.5(x+z)+0.25 y$, parameterized by the point $(b, c, d)=(0.5,0,0.25)$, starting from the initial configuration $\boldsymbol{x}=1^{4}(10)^{3}$. The simulation suggests a convergence with period $2: F^{2 t}(\boldsymbol{x}) \rightarrow$ $(\alpha 0)^{5}$ and $F^{2 t+1}(\boldsymbol{x}) \rightarrow(0 \alpha)^{5}$, for some $\alpha \in(0,1)$. As 


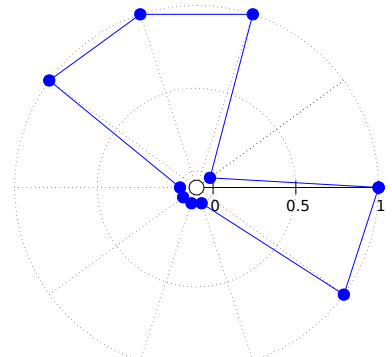

(a) $\boldsymbol{x}=101^{3} 0^{4} 1$

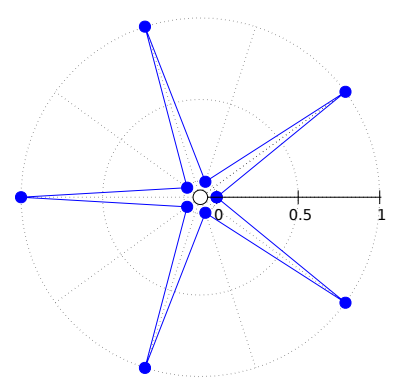

(c) $\boldsymbol{x}=(01)^{5}$

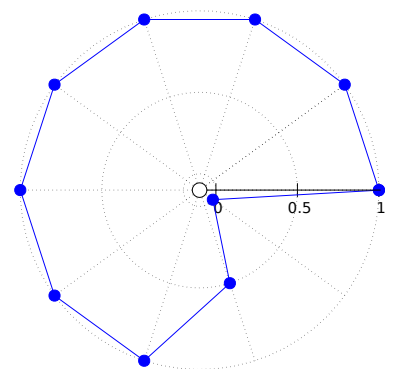

(b) $\boldsymbol{x}=1^{8} \frac{1}{2} 0$

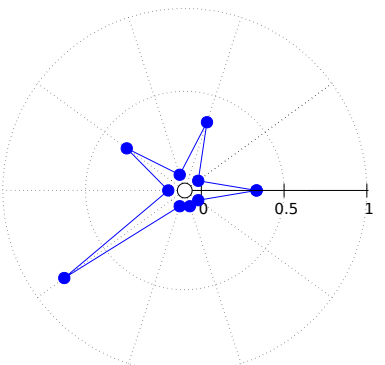

(d) $\boldsymbol{x}=\left(\frac{1}{3} 0\right)^{3} \frac{4}{5} 0^{3}$
Fig. 2: A radial view of sample configurations $\boldsymbol{x} \in$ $[0,1]^{10}$.

we will see further on, Theorem 7 will confirm this observation.

\section{Dynamical properties of legal outer-totalistic ACCAs}

In this extensive section, we provide a detailed description of the dynamics of all legal outer-totalistic ACCAs.

\subsection{General convergence results}

We start with the following simple, yet crucial observation concerning local rules parameterized by points of $\mathcal{P}$ not lying on the left face $\left(P_{0} P_{1} P_{3} P_{2}\right)$, back face $\left(P_{0} P_{5} P_{7} P_{2}\right)$ and upper face $\left(P_{2} P_{3} P_{6} P_{7}\right)$, i.e., points $(b, c, d) \in \mathcal{P}$ satisfying $b, c>0$ and $2 d-c<1$.

Lemma 1 Let $f$ be the local rule parameterized by a point $(b, c, d) \in \mathcal{P}$ and let $F$ be the corresponding global rule. Suppose that $b, c>0$ and $2 d-c<1$.

(p1) Let $(x, y, z) \in[0,1]^{3}$. Then $f(x, y, z)=0$ only for $(x, y, z)=(0,0,0)$ and, additionally, for $(x, y, z)=$ $(1,1,1)$ if $c=2 d$. Furthermore, $f(x, y, z)=1$ only for $(x, y, z)=(0,1,0)$ if $c=1$ and $(x, y, z)=(1,0,1)$ if $b=\frac{1}{2}$.

(p2) For any $\boldsymbol{x} \in X_{N}^{0,1}$ and any $t \geq 2$, the configuration $F^{t}(\boldsymbol{x})$ does not contain any 1 s.

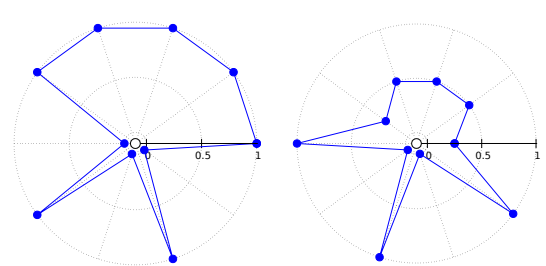

(a) $t=0$

(b) $t=1$

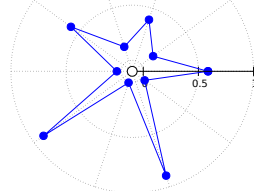

(e) $t=4$

(d) $t=3$

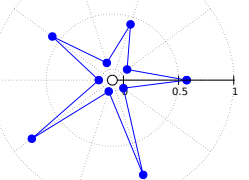

(g) $t=6$

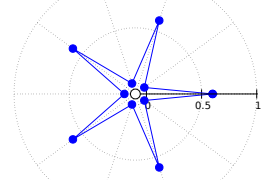

(j) $t=50$
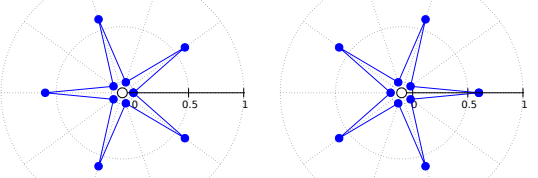

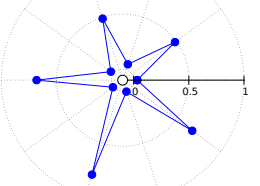

(h) $t=7$

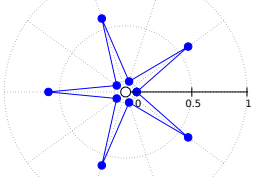

(k) $t=51$

(l) $t=100$ (n) $t=400$

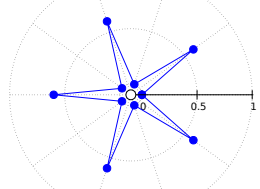

(o) $t=401$
Fig. 3: Visualization of the dynamics of an ACCA with the local rule parameterized by the point $(b, c, d)=$ $(0.5,0,0.25)$ starting from the initial configuration $\boldsymbol{x}=$ 1111101010. The subsequent radial views show $F^{t}(\boldsymbol{x})$ for given $t$.

(p3) For any $\boldsymbol{x} \in X_{N}^{0,1}$ (if $(b, c, d) \neq P_{4}$ or $N$ is odd) and for any $\boldsymbol{x} \in X_{N}^{0,1} \backslash\left\{(01)^{\frac{N}{2}},(10)^{\frac{N}{2}}\right\}$ (if $(b, c, d)=$ $P_{4}$ and $N$ is even), if $t>\frac{N}{2}+2$, then the configuration $F^{t}(\boldsymbol{x})$ does not contain any 0 s. 
(p1) Let $0<b \leq \frac{1}{2}, 0<c \leq 1$ and $c \leq 2 d<c+1$. Note that according to Eq. (4), it holds that

$$
\begin{array}{ll}
f(0,0,0)=0, & f(0,1,0)=c, \\
f(0,0,1)=f(1,0,0)=b, & f(1,0,1)=2 b, \\
f(0,1,1)=f(1,1,0)=d, & f(1,1,1)=2 d-c .
\end{array}
$$

Among the values different from $f(0,0,0)$, only $f(1,1,1)$ may be zero if $2 d-c=0$. Moreover, the vertices $(0,0,0)$ and $(1,1,1)$ do not lie on a common edge in $[0,1]^{3}$, thus it is not possible to get $f(x, y, z)=0$ if $(x, y, z)$ is neither $(0,0,0)$ nor $(1,1,1)$. This follows directly from the fact that $f$ is affine w.r.t. each variable and the minimal value of $f$ on $[0,1]^{3}$ is zero. Similarly, $f(x, y, z)$ only takes value 1 for $(0,1,0)$ if $c=1$, or $(1,0,1)$ if $b=\frac{1}{2}$.

(p2) Suppose that $\boldsymbol{x} \in X_{N}^{0,1}, t \geq 2$, but $F^{t}(\boldsymbol{x})$ contains 1 . If so, then according to (p1), $F^{t-1}(\boldsymbol{x})$ has to contain a string 01 and, consequently, $F^{t-2}(\boldsymbol{x})$ contains a string $x y z u$ such that $f(x, y, z)=0$ and $f(y, z, u)=1$. However, the first equality implies $y=z$, while the second one is possible only when $y \neq z$. This contradiction shows that $F^{t}(\boldsymbol{x})$ cannot contain any $1 \mathrm{~s}$ if $t-2 \geq 0$.

(p3) Suppose that $\boldsymbol{x} \in X_{N}^{0,1}$. From (p2) we know that $F^{2}(\boldsymbol{x})$ does not contain any $1 \mathrm{~s}$. According to (p1) if $F^{2}(\boldsymbol{x})=0^{N}$, then $F^{1}(\boldsymbol{x})=0^{N}$, or $F^{1}(\boldsymbol{x})=1^{N}$ and $c=2 d$. As the first case is impossible (since $\boldsymbol{x} \neq 0^{N}$ and $\left.\boldsymbol{x} \neq 1^{N}\right)$, it has to hold that $F^{1}(\boldsymbol{x})=1^{N}$ and $c=2 d$. However, $F^{1}(\boldsymbol{x})=1^{N}$ only when $N$ is even, $\boldsymbol{x}=(01)^{\frac{N}{2}}$ or $\boldsymbol{x}=(10)^{\frac{N}{2}}$, and both $b=\frac{1}{2}$ and $c=1$. Thus, if $(b, c, d) \neq P_{4}$ or $N$ is odd, then $F^{2}(\boldsymbol{x}) \neq 0^{N}$. The same holds when $(b, c, d)=P_{4}, N$ is even but $\boldsymbol{x} \neq(01)^{\frac{N}{2}}$ and $\boldsymbol{x} \neq(10)^{\frac{N}{2}}$. If so, then in each subsequent time step, the length of every string of 0 s in $\boldsymbol{x}$ is reduced by one on each side, hence $F^{2+s}(\boldsymbol{x})$ has no 0 for $s>\frac{N}{2}$.

Since we consider legal ACCAs, it holds that $F^{t}\left(0^{N}\right)=0^{N}$ for any $t>0$. Moreover, as we will see, for many $(b, c, d) \in \mathcal{P}$ and many $\boldsymbol{x} \in X_{N}$, it holds that $F^{t}(\boldsymbol{x}) \rightarrow 0^{N}$. The following lemma characterizes the homogeneous configurations $s^{N} \in X_{N}$ that may be the limit of some sequence $\left(F^{t}(\boldsymbol{x})\right)_{t \in \mathbb{N}}$ when $b+c-d \neq 0$.

Lemma 2 Let $f$ be the local rule parameterized by a point $(b, c, d) \in \mathcal{P}$ and let $F$ be the corresponding global rule. Suppose that $b+c-d \neq 0$. If for some $\boldsymbol{x} \in X_{N}$ it holds that $\lim _{t \rightarrow \infty} F^{t}(\boldsymbol{x})=s^{N}$, then $s=0$ or $s=$ $\frac{2 b+c-1}{2(b+c-d)}$.

Proof If $\lim _{t \rightarrow \infty} F^{t}(\boldsymbol{x})=s^{N}$, then $f(s, s, s)=s$. Hence, according to Eq. (4) we obtain

$2(d-c-b) s^{2}+(2 b+c) s=s$.

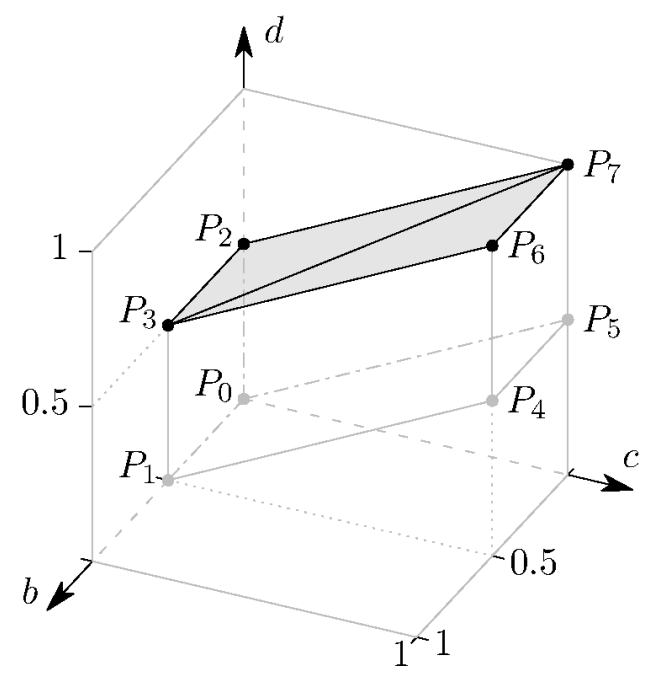

(a)

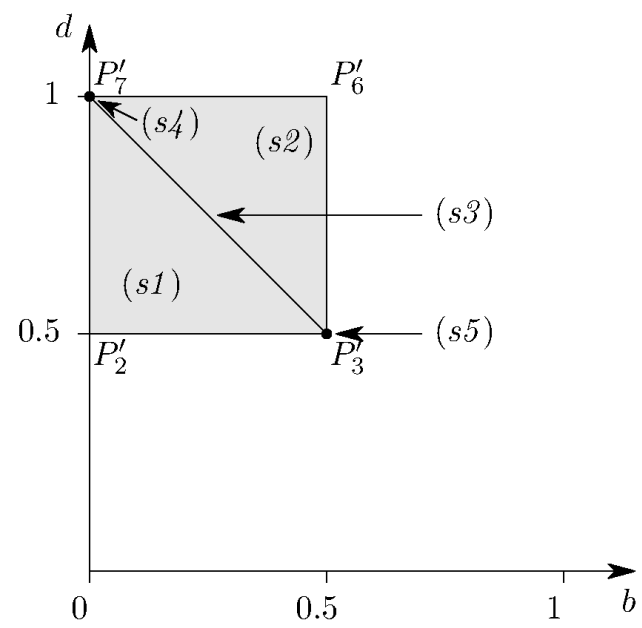

(b)

Fig. 4: Parameterization of the set of double-legal outertotalistic ACCAs: in the parallelepiped $\mathcal{P}(\mathrm{a})$ in the square $\mathcal{S}(\mathrm{b})$.

Since $b+c-d \neq 0$, Eq. (6) has only two solutions: $s=0$ or $s=\frac{2 b+c-1}{2(b+c-d)}$, which proves our claim.

Note that although $\frac{2 b+c-1}{2(b+c-d)}$ is bounded from above by 1 , it still might be negative, in which case the only possible homogeneous limit of $F^{t}(\boldsymbol{x})$ is $0^{N}$.

\subsection{The case $2 d-c=1$ : the upper face}

We start our investigation with local rules that satisfy both $l_{0}=f(0,0,0)=0$ and $l_{7}=f(1,1,1)=2 d-c=1$, i.e., double-legal local rules. These rules are parameterized by points lying on the upper face $P_{2} P_{3} P_{6} P_{7}$ of the parallelepiped $\mathcal{P}$ (see Fig. 4(a) . In this case, the 
expression of the local rule $f$ reduces to

$f(x, y, z)=(1-b-d)(x+z) y+b(x+z)+(2 d-1) y$,

where $0 \leq b \leq \frac{1}{2}$ and $\frac{1}{2} \leq d \leq 1$. Thus, the set of double-legal outer-totalistic ACCAs can also be parameterized by the points of the square $P_{2}^{\prime} P_{3}^{\prime} P_{6}^{\prime} P_{7}^{\prime}$ shown in Fig. 4(b) and denoted in the remainder by $\mathcal{S}$. Note that if $f$ is double-legal, then also $f^{C}$ is double-legal. Moreover, the points that parameterize $f$ and $f^{C}$ are symmetric w.r.t. the diagonal $P_{3}^{\prime} P_{7}^{\prime}$. As the behavior of $F^{C}$ is strongly related to the behavior of $F$, it is sufficient to consider the case $b+d \leq 1$.

In Table 3 , we list a few points $(b, c, d)$ corresponding to double-legal outer-totalistic ACCAs with distinct dynamics and indicate the corresponding groups of initial configurations identified through simulation. These distinct dynamics will be discussed analytically further on.

Table 3: Examples of points $(b, c, d)$ representing five distinct dynamics observed in the simulation of doublelegal outer-totalistic ACCAs lying on the upper face $P_{2}^{\prime} P_{3}^{\prime} P_{6}^{\prime} P_{7}^{\prime}$ of the parallelepiped $\mathcal{P}$.

\begin{tabular}{|c|l|l|}
\hline$(b, c, d)$ & \multicolumn{1}{|c|}{$N=10$} & \multicolumn{1}{|c|}{$N=11$} \\
\hline \hline$(0.2,0.4,0.7)$ & $G_{0}=X_{10}^{1}$ & $G_{0}=X_{11}^{1}$ \\
& $G_{1}=\left\{1^{10}\right\}$ & $G_{1}=\left\{1^{11}\right\}$ \\
\hline$(0.2,0.4,0.9)$ & $G_{0}=\left\{0^{10}\right\}$ & $G_{0}=\left\{0^{11}\right\}$ \\
& $G_{1}=X_{10}^{0}$ & $G_{1}=X_{11}^{0}$ \\
\hline \multirow{3}{*}{$(0.2,0.6,0.8)$} & $G_{0}=\left\{0^{10}\right\}$ & $G_{0}=\left\{0^{11}\right\}$ \\
& $G_{1}=\left\{1^{10}\right\}$ & $G_{1}=\left\{1^{11}\right\}$ \\
& $G_{2}=X_{10}^{0,1}$ & $G_{2}=X_{11}^{0,1}$ \\
\hline & $G_{0}=\left\{0^{10}\right\}$ & $G_{0}=\left\{0^{11}\right\}$ \\
$(0.0,1.0,1.0)$ & $G_{1}=\left\{1^{10}\right\}$ & $G_{1}=\left\{1^{11}\right\}$ \\
& $G_{4}=X_{10}^{0,1}$ & $G_{4}=X_{11}^{0,1}$ \\
\hline & $G_{0}=\left\{0^{10}\right\}$ & $G_{0}=\left\{0^{11}\right\}$ \\
& $G_{1}=\left\{1^{10}\right\}$ & $G_{1}=\left\{1^{11}\right\}$ \\
& $G_{2} \neq \emptyset$ & $G_{2}=X_{11}^{0,1}$ \\
& $G_{5} \neq \emptyset$ & \\
\hline
\end{tabular}

Theorem 3 Let $f$ be the local rule parameterized by a point $(b, d) \in \mathcal{S}$ and let $F$ be the corresponding global rule.

(s1) If $b+d<1$, then for any initial configuration $\boldsymbol{x} \in X_{N}^{1}$, it holds that

$\lim _{t \rightarrow \infty} F^{t}(\boldsymbol{x})=0^{N}$

while $F^{t}\left(1^{N}\right)=1^{N}$ for each $t>0$. (s2) If $b+d>1$, then for any initial configuration $\boldsymbol{x} \in X_{N}^{0}$, it holds that

$\lim _{t \rightarrow \infty} F^{t}(\boldsymbol{x})=1^{N}$,

while $F^{t}\left(0^{N}\right)=0^{N}$ for each $t>0$.

(s3) If $b+d=1$ and $0<b<\frac{1}{2}$, then for any initial configuration $\boldsymbol{x} \in X_{N}$, it holds that

$\lim _{t \rightarrow \infty} F^{t}(\boldsymbol{x})=(\rho(\boldsymbol{x}))^{N}$.

(s4) If $b=0$ and $d=1$ (i.e., $(b, d)=P_{7}^{\prime}$ ), then $f$ is the identity rule, so each configuration $\boldsymbol{x} \in X_{N}$ is a fixed point of $F$.

(s5) If $b=d=\frac{1}{2}$ (i.e., $\left.(b, d)=P_{3}^{\prime}\right)$, then for any initial configuration $\boldsymbol{x} \in X_{N}$, it holds that

- if $N$ is odd, then

$$
\lim _{t \rightarrow \infty} F^{t}(\boldsymbol{x})=(\rho(\boldsymbol{x}))^{N} \text {; }
$$

- if $N$ is even, then

$$
\left\{\begin{array}{l}
\lim _{t \rightarrow \infty} F^{2 t}(\boldsymbol{x})=\left(\rho_{0}(\boldsymbol{x}) \rho_{1}(\boldsymbol{x})\right)^{\frac{N}{2}} \\
\lim _{t \rightarrow \infty} F^{2 t+1}(\boldsymbol{x})=\left(\rho_{1}(\boldsymbol{x}) \rho_{0}(\boldsymbol{x})\right)^{\frac{N}{2}}
\end{array},\right.
$$

where $\rho_{0}(\boldsymbol{x})=\frac{2}{N}\left(x_{0}+x_{2}+\ldots+x_{N-2}\right)$ and $\rho_{1}(\boldsymbol{x})=\frac{2}{N}\left(x_{1}+x_{3}+\ldots+x_{N-1}\right)$.

Proof Consider $(b, d) \in \mathcal{S}$ and let $\boldsymbol{x} \in X_{N}^{1}$. First, let us note that if $b+d<1$, then $x, y, z \leq M \leq 1$ implies $f(x, y, z) \leq M$. Indeed,

$$
\begin{aligned}
f(x, y, z) & =(1-b-d)(x+z) y+b(x+z)+(2 d-1) y \\
& \leq(1-b-d)(x+z)+b(x+z)+(2 d-1) y \\
& \leq(1-b-d) 2 M+2 b M+(2 d-1) M=M .
\end{aligned}
$$

Moreover, from the above calculations, it is obvious that if at least one variable $x, y, z$ is strictly smaller than 1 , then also $f(x, y, z)<1$. This means that if for some $t_{0} \in \mathbb{N}$ the configuration $F^{t_{0}}(\boldsymbol{x})$ does not contain any 1s, i.e., $M=\max \left(F^{t_{0}}(\boldsymbol{x})\right)<1$, then for each $t \geq t_{0}$ and $0 \leq n \leq N-1$, it holds that $x_{n}^{t} \leq M$. Hence

$$
\begin{aligned}
\sigma\left(F^{t+1}(\boldsymbol{x})\right)= & \sum_{n=0}^{N-1} x_{n}^{t+1} \\
= & \sum_{n=0}^{N-1}\left((1-b-d)\left(x_{n-1}^{t}+x_{n+1}^{t}\right) x_{n}^{t}\right. \\
& \left.+b\left(x_{n-1}^{t}+x_{n+1}^{t}\right)+(2 d-1) x_{n}^{t}\right) \\
\leq & \left.\left(\sum_{n=0}^{N-1}(1-b-d) 2 M x_{n}^{t}\right)\right) \\
& +(2 b+2 d-1) \sigma\left(F^{t}(\boldsymbol{x})\right) \\
= & (1-b-d) 2 M \sigma\left(F^{t}(\boldsymbol{x})\right) \\
& +(2 b+2 d-1) \sigma\left(F^{t}(\boldsymbol{x})\right) \\
= & (1-2(1-b-d)(1-M)) \sigma\left(F^{t}(\boldsymbol{x})\right) .
\end{aligned}
$$


This means that $\sigma\left(F^{t}(\boldsymbol{x})\right)$ tends to zero (since $1-2(1-$ $b-d)(1-M)<1$, which concludes the proof of statement (s1). As mentioned before, statement (s2) is a direct consequence of (s1), since local rules parameterized by points $(b, d) \in \mathcal{S}$ with $b+d>1$ are conjugate to those with $b+d<1$.

If $b+d=1$, then $f(x, y, z)=b(x+z)+(1-2 b) y$ and $f$ is density-conserving, i.e., for any $\boldsymbol{x} \in X_{N}$, it holds that

$\rho(\boldsymbol{x})=\rho(F(\boldsymbol{x}))=\rho\left(F^{2}(\boldsymbol{x})\right)=\rho\left(F^{3}(\boldsymbol{x})\right)=\ldots$.

The dynamics of such ACCAs is described in [9, including the proof of statements (s3) and (s5), Statement (s4) is obvious.

4.3 The case $2 b+c<1$ and $2 d-c<1$

The points of $\mathcal{P}$ satisfying $2 b+c<1$ and $2 d-c<1$ are shown in Figure 5. The dynamics of ACCAs whose

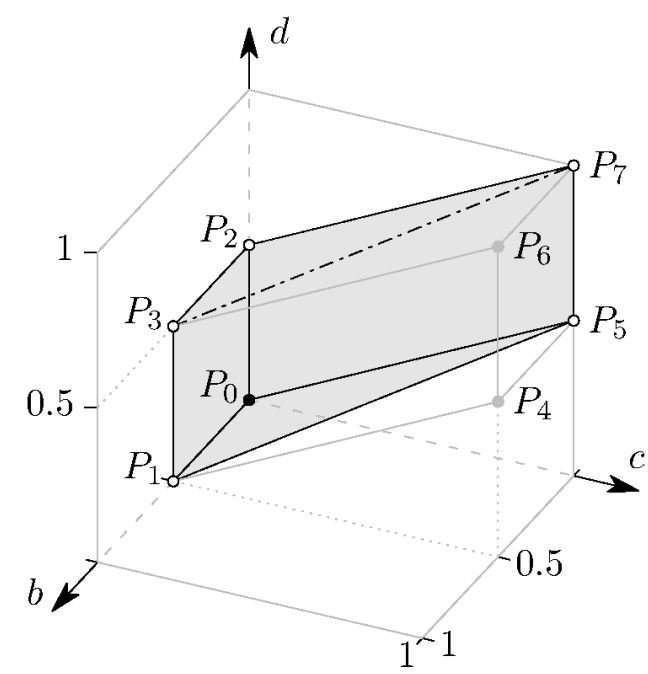

Fig. 5: Parameterization of the set of legal outertotalistic ACCAs in the case $2 b+c<1$ and $2 d-c<1$.

local rules are parameterized by these points is very simple. The state of each cell tends to zero, irrespective of the initial configuration. The following theorem formally characterizes the behavior of such CAs.

Theorem 4 Let $f$ be the local rule parameterized by a point $(b, c, d) \in \mathcal{P}$ and let $F$ be the corresponding global rule. If $2 b+c<1$ and $2 d-c<1$, then for any initial configuration $\boldsymbol{x} \in X_{N}$, it holds that $\lim _{x \rightarrow \infty} F^{t}(\boldsymbol{x})=0^{N}$.
Proof Let us choose $N \in \mathbb{N}$ and $\boldsymbol{x} \in X_{N}$. For any $t \geq 0$, it holds that

$$
\begin{aligned}
\sigma\left(F^{t+1}(\boldsymbol{x})\right)= & \sum_{n=0}^{N-1} x_{n}^{t+1} \\
= & \sum_{n=0}^{N-1}\left[(d-c-b)\left(x_{n-1}^{t}+x_{n+1}^{t}\right) x_{n}^{t}\right. \\
& \left.+b\left(x_{n-1}^{t}+x_{n+1}^{t}\right)+c x_{n}^{t}\right] \\
= & \left(\sum_{n=0}^{N-1}(d-c-b)\left(x_{n-1}^{t}+x_{n+1}^{t}\right) x_{n}^{t}\right) \\
& +(2 b+c) \sigma\left(F^{t}(\boldsymbol{x})\right) .
\end{aligned}
$$

Hence, if $d-c-b<0$, then

$\sigma\left(F^{t+1}(\boldsymbol{x})\right) \leq(2 b+c) \sigma\left(F^{t}(\boldsymbol{x})\right)$.

If $d-c-b \geq 0$, then

$$
\begin{aligned}
& \sum_{n=0}^{N-1}(d-c-b)\left(x_{n-1}^{t}+x_{n+1}^{t}\right) x_{n}^{t} \\
& \leq \sum_{n=0}^{N-1}(d-c-b)\left(x_{n-1}^{t}+x_{n+1}^{t}\right) \\
& \quad=2(d-c-b) \sigma\left(F^{t}(\boldsymbol{x})\right)
\end{aligned}
$$

thus, in this case,

$$
\begin{aligned}
\sigma\left(F^{t+1}(\boldsymbol{x})\right) & \leq 2(d-c-b) \sigma\left(F^{t}(\boldsymbol{x})\right)+(2 b+c) \sigma\left(F^{t}(\boldsymbol{x})\right) \\
& =(2 d-c) \sigma\left(F^{t}(\boldsymbol{x})\right)
\end{aligned}
$$

Since $2 b+c<1$ and $2 d-c<1$, we see that, in both cases, $\sigma\left(F^{t}(\boldsymbol{x})\right)$ tends to zero, which concludes the proof.

\subsection{The case $2 b+c \geq 1$ and $2 d-c<1$}

To conclude our investigation, we explore the dynamics of the local rules parameterized by points belonging to the polyhedron $P_{1} P_{4} P_{5} P_{3} P_{6} P_{7}$ without the upper face $P_{3} P_{6} P_{7}$, denoted by $\mathcal{P}_{0}$ and shown in Figure 6 . This set is explicitly given as

$\mathcal{P}_{0}=\{(b, c, d) \in \mathcal{P} \mid 2 b+c \geq 1$ and $2 d-c<1\}$.

In this subsection, the expression $\frac{2 b+c-1}{2(b+c-d)}$ will be denoted by $\lambda$ and will turn out to be a key parameter. Note that if $(b, c, d) \in \mathcal{P}_{0}$, then it holds in particular that $b+c-d>0$, hence $\lambda$ is well defined and lies in $[0,1]$.

This section is organized as follows. We start with some technical lemmata. Then we describe the dynamics of the rules parameterized by points belonging to $\mathcal{P}_{0}$, except for the three edges $P_{1} P_{3}, P_{4} P_{6}$ and $P_{5} P_{7}$. 


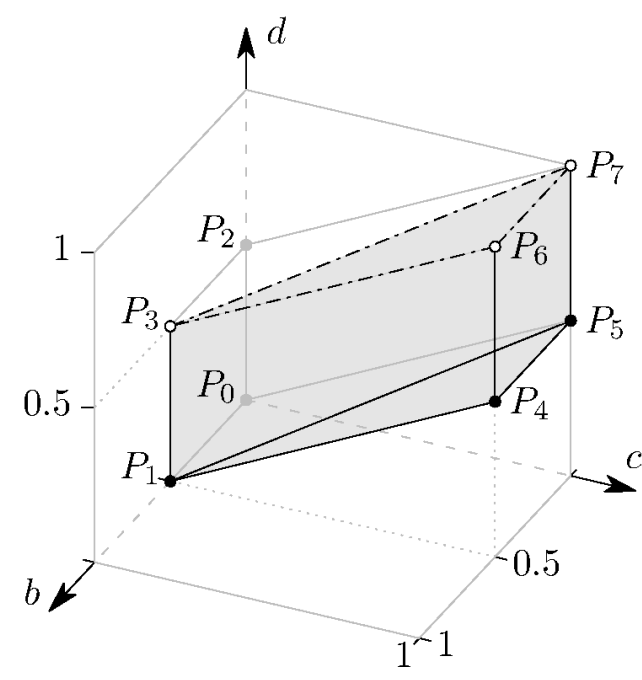

Fig. 6: The polyhedron $\mathcal{P}_{0}$.

Next, we consider the edges $P_{4} P_{6}, P_{1} P_{3}$ and $P_{5} P_{7}$ one by one. The latter case is the most complicated one, since the rules parameterized by points from $P_{5} P_{7}$ exhibit the most surprising behavior.

\subsubsection{Some technical lemmata}

In order to prove the main theorems in this section, we introduce a few lemmata.

Lemma 3 Let $f$ be the local rule parameterized by a point $(b, c, d) \in \mathcal{P}_{0}$. The function $g:[0,1] \rightarrow \mathbb{R}$ defined by

$g(s):=f(s, s, s)=-2(b+c-d) s^{2}+(2 b+c) s$

has the following two properties;

(g1) Let $s_{\star} \in[\lambda, 1]$. If $0 \leq s \leq s_{\star}$, then $0 \leq g(s) \leq s_{\star}$.

(g2) If $0<s<1$, then $\lim _{t \rightarrow \infty} g^{t}(s)=\lambda$, where $g^{t}(s)=\underbrace{g \circ g \circ \ldots \circ g}_{t \text { times }}(s)$.

Proof Assume first that $2 b+c>1$, then the graph of the function $g$ is shown in Figure $7(\mathrm{a})$ (under the proviso that $s_{v}$ may lie to the right of 1 or $s_{2}$ may be equal to 1). Indeed, the function $g$ is a quadratic function with roots $s_{1}=0$ and $s_{2}=\frac{2 b+c}{2(b+c-d)}$, restricted to the domain $[0,1]$. It has two fixed points, namely 0 and $\lambda$. Since $c \leq 2 d \leq c+1$, it holds that $\lambda \leq 1 \leq s_{2}$. Directly from the graph of $g$, we can conclude that (g1) holds, since for $s \geq \lambda$ we have $g(s) \leq s$. Moreover, the fixed point $s_{1}=0$ is a repeller, while $\lambda$ is an attractor with basin including $(0,1)$. This implies (g2)

Next, assume that $2 b+c=1$. In that case, it holds that $\lambda=0$ and the line $y=s$ is tangent to the graph

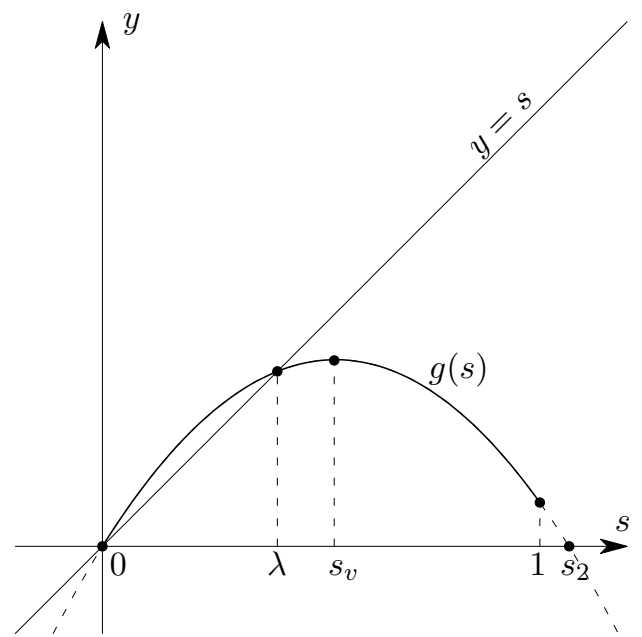

(a)

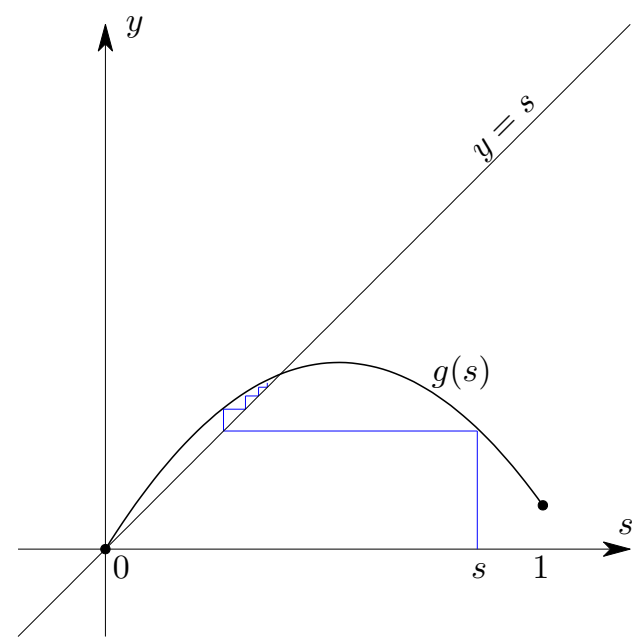

(b)

Fig. 7: The graph of the function $g$ in the case $2 b+c>1$. Location of the characteristic points (a) and attractor for an example point $s \in(0,1)(\mathrm{b})$

of $g$ at $s_{1}=0$ (see Figure 8) and one can see that 0 is an attractor with basin $[0,1]$.

As a simple consequence of Lemma 3, we get the following description of the orbits for the initial configuration $1^{N}$.

Lemma 4 Let $f$ be the local rule parameterized by a point $(b, c, d) \in \mathcal{P}_{0}$ and let $F$ be the corresponding global rule. If $c=2 d$ (i.e., $(b, c, d)$ belongs to the lower face $\left.P_{1} P_{4} P_{5}\right)$, then $F^{t}\left(1^{N}\right)=0^{N}$, for each $t \geq 1$; otherwise, $F^{t}\left(1^{N}\right) \stackrel{t \rightarrow \infty}{\longrightarrow} \lambda^{N}$.

Proof Note that $f(1,1,1)=2 d-c$. If $2 d=c$, then it holds that $F\left(1^{N}\right)=0^{N}$, whence $F^{t}\left(1^{N}\right)=0^{N}$, for any $t \geq 1$. If $2 d \neq c$, then it holds that $2 d-c \in(0,1)$ 


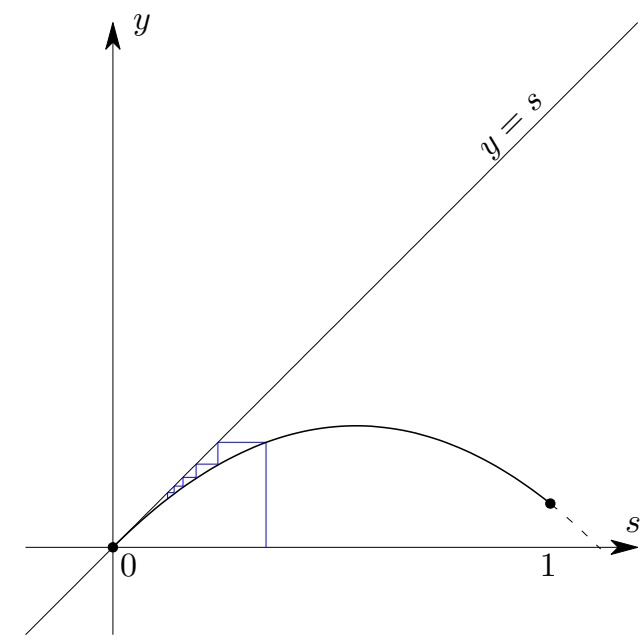

Fig. 8: The graph of the function $g$ in the case $2 b+c=1$. The line $y=s$ is tangent to the graph of $g$ at $s_{1}=0$.

(since $\left.(b, c, d) \in \mathcal{P}_{0}\right)$. Taking into account that $g(s)=$ $f(s, s, s)$, it then follows that

$F^{t}\left(1^{N}\right)=\left(g^{t-1}(2 d-c)\right)^{N} \stackrel{t \rightarrow \infty}{\longrightarrow} \lambda^{N}$,

according to property (g2) of Lemma 3

Since the behavior of $F$ on $1^{N}$ is now understood, in the remainder we will only consider initial configurations $\boldsymbol{x}$ from $X_{N}^{0,1}$.

Lemma 5 Let $f$ be the local rule parameterized by a point $(b, c, d) \in \mathcal{P}_{0}$ and let $F$ be the corresponding global rule. For each $\varepsilon>0$, for each $t_{0} \in \mathbb{N}$ and for any initial configuration $\boldsymbol{x} \in X_{N}$, there exists $t_{\varepsilon} \geq t_{0}$ such that

$\min \left(F^{t_{\varepsilon}}(\boldsymbol{x})\right)<\lambda+\varepsilon$.

Proof We give a proof by contradiction. Let $\varepsilon>0$ and $t_{0} \in \mathbb{N}$ be given and assume that for some initial configuration $\boldsymbol{x}$ it holds for each $t \geq t_{0}$ that

$\min \left(F^{t}(\boldsymbol{x})\right) \geq \lambda+\varepsilon$.

For every $t \geq t_{0}$ and any $n \in\{0,1, \ldots, N-1\}$, it holds that

$$
\begin{aligned}
x_{n}^{t+1}= & f\left(x_{n-1}^{t}, x_{n}^{t}, x_{n+1}^{t}\right) \\
= & -(b+c-d)\left(x_{n-1}^{t}+\right. \\
& \left.x_{n+1}^{t}\right) x_{n}^{t}+b\left(x_{n-1}^{t}+x_{n+1}^{t}\right)+c x_{n}^{t} \\
\leq & -(b+c-d)\left(x_{n-1}^{t}+x_{n+1}^{t}\right)(\lambda+\varepsilon) \\
& +b\left(x_{n-1}^{t}+x_{n+1}^{t}\right)+c x_{n}^{t} \\
= & \left(\frac{1}{2}-\frac{1}{2} c-(b+c-d) \varepsilon\right)\left(x_{n-1}^{t}+x_{n+1}^{t}\right)+c x_{n}^{t},
\end{aligned}
$$

since $b+c-d>0, x_{n}^{t} \geq \lambda+\varepsilon$ and $\lambda=\frac{2 b+c-1}{2(b+c-d)}$. Hence, for each $t \geq t_{0}$ we find

$$
\begin{aligned}
0 & \leq \sigma\left(F^{t+1}(\boldsymbol{x})\right) \\
& \leq \sum_{n=0}^{N-1}\left[\left(\frac{1}{2}-\frac{1}{2} c-(b+c-d) \varepsilon\right)\left(x_{n-1}^{t}+x_{n+1}^{t}\right)+c x_{n}^{t}\right] \\
& =(1-2(b+c-d) \varepsilon) \sigma\left(F^{t}(\boldsymbol{x})\right) .
\end{aligned}
$$

Note that $1-2(b+c-d) \varepsilon<1$, so the above inequality implies $\sigma\left(F^{t}(\boldsymbol{x})\right) \stackrel{t \rightarrow \infty}{\longrightarrow} 0$, contrary to the assumption in Eq. (7).

The following lemma lists some dependencies between the elements of the set $\mathcal{V}$ introduced in Theorem 2 . Note that if $f$ is a local rule defined by Eq. (4), then the set $\mathcal{V}$ contains at most six different numbers:

$$
\begin{aligned}
V_{1}=f(m, m, m)= & -2(b+c-d) m^{2}+(2 b+c) m ; \\
V_{2}=f(M, m, m)= & -(b+c-d) M m-(b+c-d) m^{2} \\
& +b M+(b+c) m ; \\
V_{3}=f(m, M, m)= & -2(b+c-d) M m+2 b m+c M ; \\
V_{4}=f(M, m, M)= & -2(b+c-d) M m+2 b M+c m ; \\
V_{5}=f(M, M, m)= & -(b+c-d) M m-(b+c-d) M^{2} \\
& +b m+(b+c) M ; \\
V_{6}=f(M, M, M)= & -2(b+c-d) M^{2}+(2 b+c) M .
\end{aligned}
$$

Moreover, $V_{2}$ always lies between $V_{1}$ and $V_{4}$, while $V_{5}$ always lies between $V_{3}$ and $V_{6}$ (since $f$ is affine also w.r.t. $x+z$ ). The following lemma presents some dependencies between $V_{1}, V_{3}, V_{4}$ and $V_{6}$.

Lemma 6 Let $f$ be the local rule parameterized by a point $(b, c, d) \in \mathcal{P}_{0}$ and let $0 \leq m<M \leq 1$. Then it holds that

(d1) $V_{1} \leq V_{4}$ if and only if $m \leq \frac{b}{b+c-d}$.

(d2) $V_{3} \leq V_{6}$ if and only if $M \leq \frac{b}{b+c-d}$.

(d3) $V_{1} \leq V_{3}$ if and only if $m \leq \frac{b+c}{2(b+c-d)}$.

(d4) $V_{4} \leq V_{3}$ if and only if $c \geq 2 b$.

(d5) $V_{4} \leq V_{6}$ if and only if $M \leq \frac{c}{2(b+c-d)}$.

Proof Statements (d1) (d5) respectively follow from

$V_{4}-V_{1}=2(M-m)(b-(b+c-d) m)$,

$V_{6}-V_{3}=2(M-m)(b-(b+c-d) M)$,

$V_{3}-V_{1}=(M-m)(c-2(b+c-d))$,

$V_{3}-V_{4}=(M-m)(c-2 b)$,

$V_{4}-V_{6}=(M-m)(2(b+c-d) M-c)$,

which concludes the proof.

Equipped with the above facts, we study the behavior of ACCAs parameterized by points in $\mathcal{P}_{0}$. We divide $\mathcal{P}_{0}$ into several parts, because for each part we use different proof methods. 
4.4.2 The case $(b, c, d) \in \mathcal{P}_{0} \backslash\left(P_{1} P_{3} \cup P_{4} P_{6} \cup P_{5} P_{7}\right)$.

In this subsection, we uncover the dynamics of local rules parameterized by points $(b, c, d)$ that belong to $\mathcal{P}_{0}$ but do not lie on any of the edges $P_{1} P_{3}, P_{4} P_{6}$ and $P_{5} P_{7}$, i.e., $0<b<\frac{1}{2}$ or $0<c<1$. In this case, the dynamics turns out to be very simple: each initial configuration $\boldsymbol{x} \in X_{N}^{0,1}$ tends to the same homogeneous one, as stated in the following theorem.

Theorem 5 Let $f$ be the local rule parameterized by a point $(b, c, d) \in \mathcal{P}_{0}$ and let $F$ be the corresponding global rule. If $0<b<\frac{1}{2}$ or $0<c<1$, then for any initial configuration $\boldsymbol{x} \in X_{N}^{0,1}$, it holds that

$\lim _{t \rightarrow \infty} F^{t}(\boldsymbol{x})=\lambda^{N}$.

Proof Let $(b, c, d) \in \mathcal{P}_{0}$, where $0<b<\frac{1}{2}$ or $0<c<1$, and $\boldsymbol{x} \in X_{N}^{0,1}$. We consider the case $c \geq 2 b$, the case $c<2 b$ being analogous. Suppose that $\bar{b}=\frac{1}{2}$, then it would also hold that $c=1$, a contradiction. Hence, it holds that $b<\frac{1}{2}$, which implies that $\varepsilon=\frac{1-2 b}{2(b+c-d)}>0$. Since $2 d-c<1$, according to Lemma 1.(p2) (p3) and Lemma 5 , there exists $t_{\varepsilon} \geq 2$ such that $0<m_{0}<\lambda+\varepsilon$ and $M_{0}<1$, where

$m_{0}=\min \left(F^{t_{\varepsilon}}(\boldsymbol{x})\right)$ and $M_{0}=\max \left(F^{t_{\varepsilon}}(\boldsymbol{x})\right)$.

If $m_{0}=M_{0}$, i.e., $F^{t_{\varepsilon}}(\boldsymbol{x})$ is a homogeneous configuration $\left(m_{0}\right)^{N}$, then according to Lemma 3 , it holds that $F^{t}(\boldsymbol{x})$ tends to $\lambda^{N}$. Therefore, we can assume that $m_{0}<M_{0}$.

Consider the following two sequences $\left(m_{t}\right)_{t>0}$ and $\left(M_{t}\right)_{t \geq 0}$ :

$m_{t+1}=\min \left\{f(x, y, z) \mid x, y, z \in\left\{m_{t}, M_{t}\right\}\right\}$,

$M_{t+1}=\max \left\{f(x, y, z) \mid x, y, z \in\left\{m_{t}, M_{t}\right\}\right\}$.

According to Theorem 2 it holds that

$F^{t_{\varepsilon}+t}(\boldsymbol{x}) \in\left[m_{t}, M_{t}\right]^{N}$.

We will show that the sequence of $N$-dimensional cubes $\left[m_{t}, M_{t}\right]^{N}$ collapses to a single point $\lambda^{N}$. We consider two cases.

Case one. Assume that for some $t_{0} \geq 0$, it holds that $M_{t_{0}} \leq \frac{b}{b+c-d}$. Then obviously $m_{t_{0}} \leq \frac{b}{b+c-d}$ and from Lemma 6 it follows that

$M_{t_{0}+1}=f\left(M_{t_{0}}, M_{t_{0}}, M_{t_{0}}\right)=g\left(M_{t_{0}}\right)$

and

$m_{t_{0}+1}=f\left(m_{t_{0}}, m_{t_{0}}, m_{t_{0}}\right)=g\left(m_{t_{0}}\right)$.

Note that $c \geq 2 b$ implies that $\lambda \leq \frac{b}{b+c-d} \leq s_{v}$. Thus,

$M_{t_{0}+1}=g\left(M_{t_{0}}\right) \leq g\left(\frac{b}{b+c-d}\right) \leq \frac{b}{b+c-d}$.
By simple induction we get $M_{t} \leq \frac{b}{b+c-d}$ for $t \geq t_{0}$ and consequently $M_{t+1}=g\left(M_{t}\right)$ and $m_{t+1}=g\left(m_{t}\right)$. Therefore, according to Lemma 3/(g2), we have

$\lim _{t \rightarrow \infty} M_{t}=\lim _{t \rightarrow \infty} g\left(M_{t}\right)=\lim _{t \rightarrow \infty} g^{t}\left(M_{t_{0}}\right)=\lambda$

and

$\lim _{t \rightarrow \infty} m_{t}=\lim _{t \rightarrow \infty} g\left(m_{t}\right)=\lim _{t \rightarrow \infty} g^{t}\left(m_{t_{0}}\right)=\lambda$,

which concludes the proof in the first case.

Case two. Assume that for all $t \geq 0$, it holds that $M_{t}>\frac{b}{b+c-d}$. Let us note that for all $t \geq 0$ it holds that $m_{t}<\lambda+\varepsilon$. Indeed, from Lemma 3.(g1), it follows that $m_{t+1} \leq f\left(m_{t}, m_{t}, m_{t}\right)=g\left(m_{t}\right)<\lambda+\varepsilon=\frac{c}{2(b+c-d)}$

It follows from Lemma $6(\mathrm{~d} 2),(\mathrm{d} 3)$ and $(\mathrm{d} 4)$ that for all $t \geq 0$ it holds that

$$
\begin{aligned}
M_{t+1} & =f\left(m_{t}, M_{t}, m_{t}\right) \\
& =c M_{t}+2 m_{t}\left(b-(b+c-d) M_{t}\right) \leq c M_{t},
\end{aligned}
$$

which is only possible for $c=1$, as otherwise the sequence $\left(M_{t}\right)_{t=0}^{\infty}$ would decrease exponentially to zero, contradicting the assumption that $M_{t}>\frac{b}{b+c-d}>0$, for all $t \geq 0$. Note that if $c=1$, then $\frac{b}{b+c-d}=\lambda<M_{t}$ and the sequence $\left(M_{t}\right)_{t=0}^{\infty}$ is decreasing. Again, we consider two cases.

(a) Assume that for all $t \geq 0$, it holds that also $m_{t} \geq \lambda$. It then holds that $\lambda \leq m_{t} \leq M_{t}$. Using Eq. (8), it follows that

$$
\begin{aligned}
M_{t+1}-\lambda & =M_{t}+2 m_{t}(b+1-d)\left(\lambda-M_{t}\right)-\lambda \\
& =\left(M_{t}-\lambda\right)\left(1-2 m_{t}(b+1-d)\right) \\
& \leq(1-2 b)\left(M_{t}-\lambda\right) .
\end{aligned}
$$

Since $0<b<\frac{1}{2}$, the above upper bound shows that the difference between $M_{t}$ and $\lambda$ decreases exponentially to 0 , i.e., $\lim _{t \rightarrow \infty} M_{t}=\lambda$, which implies that also $\lim _{t \rightarrow \infty} m_{t}=\lambda$.

(b) Assume that for some $\tau \geq 0$, it holds that $m_{\tau}<\lambda$. Of course, then due to Lemma 3 (g1), it holds that $m_{t} \leq \lambda$ for all $t \geq \tau$. It follows from Lemma $6(\mathrm{~d} 1)$ and (d2) that for all $t \geq \tau$, it holds that

$m_{t+1}=\min \left(g\left(m_{t}\right), g\left(M_{t}\right)\right)$.

Let $\delta=\min \left(m_{\tau}, g\left(M_{\tau}\right)\right)$. On the one hand, it holds that $0<m_{\tau} \leq \lambda$. On the other hand, $\lambda<M_{\tau}<1$ implies $g\left(M_{\tau}\right)>0$. This yields $0<\delta \leq \lambda$. We will show that $m_{t} \geq \delta$ for all $t \geq \tau$ (it holds for $t=\tau)$. The following reasoning is based on Figure $7(\mathrm{a})$. Since $\lambda<M_{t} \leq M_{\tau}$, it holds that $g\left(M_{t}\right) \geq$ $\min \left(\lambda, g\left(M_{\tau}\right)\right) \geq \delta$. Moreover, since $m_{t} \leq \lambda$, it holds that $g\left(m_{t}\right) \geq m_{t} \geq \delta$. Together, according 
to Eq. (9), we obtain $m_{t+1} \geq \delta$.

Now, let us compare the differences $M_{t+1}-m_{t+1}$ and $M_{t}-m_{t}$ for $t \geq \tau$. If $m_{t+1}=g\left(M_{t}\right)$, then it follows from Eq. (8) and Lemma 3 that

$$
\begin{aligned}
M_{t+1}-m_{t+1} & =\left(M_{t}-m_{t}\right)\left(2(b+1-d) M_{t}-2 b\right) \\
& \leq\left(M_{t}-m_{t}\right) 2(1-d) M_{t} \\
& \leq\left(M_{t}-m_{t}\right) 2(1-d) M_{\tau} .
\end{aligned}
$$

Similarly, if $m_{t+1}=g\left(m_{t}\right)$, then

$$
\begin{aligned}
M_{t+1}-m_{t+1} & =\left(M_{t}-m_{t}\right)\left(1-2(b+1-d) m_{t}\right) \\
& \leq\left(M_{t}-m_{t}\right)(1-2(b+1-d) \delta) .
\end{aligned}
$$

In both cases, we have

$$
M_{t+1}-m_{t+1} \leq \alpha\left(M_{t}-m_{t}\right)
$$

for all $t \geq \tau$, where

$$
\alpha=\max \left(2(1-d) M_{\tau}, 1-2(b+1-d) \delta\right),
$$

Note that $c=1$ implies that $\frac{1}{2} \leq d<1$. Together with $\delta>0$ and $b<\frac{1}{2}$, it then follows that $\alpha<1$. This means hat

$\lim _{t \rightarrow \infty} m_{t}=\lim _{t \rightarrow \infty} M_{t}=\lambda$

as $m_{t} \leq \lambda \leq M_{t}$, for all $t \geq \tau$.

\subsubsection{The case $(b, c, d)=\left(\frac{1}{2}, 1, d\right)$ for $\frac{1}{2} \leq d<1$.}

In this subsection, we discuss the dynamics of local rules parameterized by points belonging to the edge $P_{4} P_{6}$. In this case, $\lambda=\frac{1}{3-2 d}$ and the local rule is given by

$f(x, y, z)=-\left(\frac{3}{2}-d\right)(x+z) y+\frac{1}{2}(x+z)+y$.

An interesting observation is the following bifurcation: for any even $N \in \mathbb{N}$, it holds that

$\lim _{t \rightarrow \infty} F^{t}\left((01)^{\frac{N}{2}}\right)=\lambda^{N}$ if $\frac{1}{2}<d<1$, and

$\lim _{t \rightarrow \infty} F^{t}\left((01)^{\frac{N}{2}}\right)=0^{N}$ if $d=\frac{1}{2}$, while

$\lim _{d \rightarrow \frac{1}{2}^{+}} \lambda=\lim _{d \rightarrow \frac{1}{2}^{+}} \frac{1}{3-2 d}=\frac{1}{2} \neq 0$.

Theorem 6 Let $f$ be the local rule parameterized by a point $\left(\frac{1}{2}, 1, d\right) \in \mathcal{P}_{0}$ and let $F$ be the corresponding global rule. If $\frac{1}{2}<d<1$, then for any initial configuration $\boldsymbol{x} \in X_{N}^{0,1}$, it holds that

$\lim _{t \rightarrow \infty} F^{t}(\boldsymbol{x})=\lambda^{N}$.

If $d=\frac{1}{2}$, then
- if $N$ is odd, then for any $\boldsymbol{x} \in X_{N}^{0,1}$, it holds that

$\lim _{t \rightarrow \infty} F^{t}(\boldsymbol{x})=\lambda^{N}=\left(\frac{1}{2}\right)^{N} ;$

- if $N$ is even, then for any $\boldsymbol{x} \in X_{N}^{0,1} \backslash\left\{(01)^{\frac{N}{2}},(10)^{\frac{N}{2}}\right\}$, it holds that

$\lim _{t \rightarrow \infty} F^{t}(\boldsymbol{x})=\lambda^{N}=\left(\frac{1}{2}\right)^{N}$,

while $F^{t}\left((01)^{\frac{N}{2}}\right)=F^{t}\left((10)^{\frac{N}{2}}\right)=0^{N}$ for $t \geq 2$.

Proof Let $\boldsymbol{x} \in X_{N}^{0,1}$. For any $t \geq 0$, let $\mu_{t}$ denote the maximal absolute difference between $x_{n}^{t}$ and $\lambda$, i.e., $\mu_{t}=\max _{0 \leq n \leq N-1}\left|x_{n}^{t}-\lambda\right|$. As $\frac{1}{2} \leq \lambda<1$, it holds that $\mu_{t} \leq \lambda$. Additionally,

$f(x, y, z)-\lambda=(\lambda-y)\left(\frac{3}{2}-d\right)(x-\lambda+z-\lambda)$,

implies

$|f(x, y, z)-\lambda| \leq|y-\lambda|\left(\frac{3}{2}-d\right)(|x-\lambda|+|z-\lambda|)$,

which means that

$\mu_{t+1} \leq \mu_{t}\left(\frac{3}{2}-d\right) 2 \mu_{t}=(3-2 d) \mu_{t}^{2}$.

In particular, since $\mu_{t} \leq \lambda=\frac{1}{3-2 d}$, it holds that $\mu_{t+1} \leq$ $\mu_{t}$.

Lemma 11(p2) implies that there exists $\tau \geq 2$ such that $m=\min \left(F^{\tau}(\boldsymbol{x})\right)>0$. Similarly, Lemma 11(p3) implies that there exists some $\tau>\frac{N}{2}+2$ such that $M=\max \left(F^{\tau}(\boldsymbol{x})\right)<1$ in the following cases: (i) $\frac{1}{2}<$ $d<1$, (ii) $d=\frac{1}{2}$ and $N$ is odd, or (iii) $d=\frac{1}{2}, N$ is even and $\boldsymbol{x} \in X_{N}^{0,1} \backslash\left\{(01)^{\frac{N}{2}},(10)^{\frac{N}{2}}\right\}$. Hence, there exists some $\tau>0$ such that

$m=\min \left(F^{\tau}(\boldsymbol{x})\right)>0 \quad$ and $\quad M=\max \left(F^{\tau}(\boldsymbol{x})\right)<1$.

This implies that $\mu_{\tau}$ is strictly smaller than $\lambda$. Indeed, $\mu_{\tau}=\max _{0 \leq n \leq N-1}\left|x_{n}^{\tau}-\lambda\right|=\max (|M-\lambda|,|m-\lambda|)<\lambda$.

Therefore, according to Eq. 10, for any $t \geq \tau$ it holds that

$\mu_{t+1} \leq\left((3-2 d) \mu_{\tau}\right) \mu_{t}$,

where $(3-2 d) \mu_{\tau}<1$. As a consequence, we get that $\mu_{t} \stackrel{t \rightarrow \infty}{\longrightarrow} 0$, which means that $\lim _{t \rightarrow \infty} F^{t}(\boldsymbol{x})=\lambda^{N}$.

Note that if $d=\frac{1}{2}$, then $\lambda=\frac{1}{2}$ and

$f(x, y, z)=-(x+z) y+\frac{1}{2}(x+z)+y$.

One easily verifies that in this case

$F^{t}\left((01)^{\frac{N}{2}}\right)=F^{t}\left((10)^{\frac{N}{2}}\right)=0^{N}$

for each $t \geq 2$. 
4.4.4 The case $(b, c, d)=\left(\frac{1}{2}, 0, d\right)$ for $0 \leq d<\frac{1}{2}$.

In this subsection, we discuss the dynamics of local rules parameterized by points belonging to the edge $P_{1} P_{3}$. In this case, the local rule is given by

$f(x, y, z)=\frac{1}{2}(x+z)(1-(1-2 d) y)$,

which yields

$f(x, y, z) \leq \frac{1}{2}(x+z) \leq \max (x, z)$.

Moreover, the alternating sum of states, i.e.,

$(-1)^{t} x_{0}^{t}+(-1)^{t+1} x_{1}^{t}+(-1)^{t+2} x_{2}^{t}+\ldots+(-1)^{t+N-1} x_{N-1}^{t}$,

is an invariant of this dynamical system. Indeed,

$$
\begin{aligned}
\sum_{j=0}^{N-1}(-1)^{t+1+j} x_{j}^{t+1}= & \sum_{j=0}^{N-1}(-1)^{t+1+j}\left[\frac{1}{2} x_{j-1}^{t}+\frac{1}{2} x_{j+1}^{t}+\right. \\
& \left.-\frac{1}{2}(1-2 d)\left(x_{j-1}^{t} x_{j}^{t}+x_{j}^{t} x_{j+1}^{t}\right)\right] .
\end{aligned}
$$

Due to the periodic boundary conditions, we have

$\sum_{j=0}^{N-1}(-1)^{j} x_{j-1}^{t}=\sum_{j=0}^{N-1}(-1)^{j} x_{j+1}^{t}=\sum_{j=0}^{N-1}(-1)^{j+1} x_{j}^{t}$

and

$$
\begin{aligned}
\sum_{j=0}^{N-1}(-1)^{j} x_{j-1}^{t} x_{j}^{t} & =\sum_{j=0}^{N-1}(-1)^{j+1} x_{j}^{t} x_{j+1}^{t} \\
& =-\sum_{j=0}^{N-1}(-1)^{j} x_{j}^{t} x_{j+1}^{t},
\end{aligned}
$$

which implies

$\sum_{j=0}^{N-1}(-1)^{t+1+j} x_{j}^{t+1}=\sum_{j=0}^{N-1}(-1)^{t+j} x_{j}^{t}$.

The following theorem describes the dynamics of such CAs.

Theorem 7 Let $f$ be the local rule parameterized by a point $\left(\frac{1}{2}, 0, d\right) \in \mathcal{P}_{0}$, where $0 \leq d<\frac{1}{2}$, and let $F$ be the corresponding global rule.

(i) If $N$ is even, then for any $\boldsymbol{x} \in X_{N}$, it holds that

$$
\begin{aligned}
& \lim _{t \rightarrow \infty} F^{2 t}(\boldsymbol{x})=(\alpha 0)^{\frac{N}{2}} \text { and } \lim _{t \rightarrow \infty} F^{2 t+1}(\boldsymbol{x})=(0 \alpha)^{\frac{N}{2}} \\
& \text { if } \alpha=\frac{2}{N}\left(x_{0}-x_{1}+x_{2}-x_{3}+\ldots-x_{N-1}\right) \geq 0, \text { while } \\
& \lim _{t \rightarrow \infty} F^{2 t}(\boldsymbol{x})=(0|\alpha|)^{\frac{N}{2}} \text { and } \lim _{t \rightarrow \infty} F^{2 t+1}(\boldsymbol{x})=(|\alpha| 0)^{\frac{N}{2}} \\
& \text { if } \alpha<0 .
\end{aligned}
$$

(ii) If $N$ is odd, then for any $\boldsymbol{x} \in X_{N}$, it holds that

$$
\lim _{t \rightarrow \infty} F^{t}(\boldsymbol{x})=0^{N} .
$$

Proof Let $\boldsymbol{x} \in X_{N}$. First, we consider even $N$. For each $t \geq 0$, we consider the following two sets

$$
\begin{aligned}
E_{t} & =\{j \in\{0,1, \ldots, N-1\} \mid j \equiv t(\bmod 2)\} \\
O_{t} & =\{j \in\{0,1, \ldots, N-1\} \mid j \not \equiv t(\bmod 2)\} \\
& =\{0,1, \ldots, N-1\} \backslash E_{t} .
\end{aligned}
$$

Note that

$E_{0}=E_{2}=E_{4}=\ldots=\{0,2, \ldots, N-2\}$,

$E_{1}=E_{3}=E_{5}=\ldots=\{1,3, \ldots, N-1\}$,

$O_{0}=O_{2}=O_{4}=\ldots=\{1,3, \ldots, N-1\}$,

$O_{1}=O_{3}=O_{5}=\ldots=\{0,2, \ldots, N-2\}$.

Let $\alpha_{t}=\max _{j \in E_{t}} x_{j}^{t}$ and $\beta_{t}=\max _{j \in O_{t}} x_{j}^{t}$. According to Eq. 12, for any $t \geq 0$ it holds that $\alpha_{t+1} \leq \alpha_{t}$ and $\beta_{t+1} \leq \beta_{t}$. This means that both sequences $\left(\alpha_{t}\right)_{t \geq 0}$ and $\left(\beta_{t}\right)_{t \geq 0}$ are convergent. Hence, $\alpha=\lim _{t \rightarrow \infty} \alpha_{t}$ and $\beta=$ $\lim _{t \rightarrow \infty} \beta_{t}$. Now consider $\delta>0$, then there exists $\tau \geq 0$ such that for any $t \geq \tau$, it holds that $\alpha \leq \alpha_{t}<\alpha+\delta$. We will show that for any $t \geq \tau$ and any $j \in E_{t}$, it holds that

$x_{j}^{t} \geq \alpha-\left(2^{\frac{N}{2}-1}-1\right) \delta$.

Indeed, suppose that this is not true, i.e., there exist $t_{0} \geq \tau$ and $j_{0} \in E_{t_{0}}$ such that $x_{j_{0}}^{t_{0}}<\alpha-\varepsilon$, where $\varepsilon=\left(2^{\frac{N}{2}-1}-1\right) \delta$. According to Eq. 12 we have

$$
\begin{aligned}
x_{j_{0}-1}^{t_{0}+1}, x_{j_{0}+1}^{t_{0}+1} & <\frac{1}{2}(\alpha+\delta+\alpha-\varepsilon)=\alpha-\frac{1}{2} \varepsilon+\frac{1}{2} \delta \\
x_{j_{0}-2}^{t_{0}+2}, x_{j_{0}}^{t_{0}+2}, x_{j_{0}+2}^{t_{0}+2} & <\frac{1}{2}\left(\alpha+\delta+\alpha-\frac{1}{2} \varepsilon+\frac{1}{2} \delta\right) \\
& =\alpha-\frac{1}{4} \varepsilon+\frac{3}{4} \delta
\end{aligned}
$$

and so on. Thus, after $\frac{N}{2}-1$ steps, we get that for each $j \in E_{t_{0}+\frac{N}{2}-1}$

$x_{j}^{t_{0}+\frac{N}{2}-1}<\alpha-\frac{1}{2^{\frac{N}{2}-1}} \varepsilon+\frac{2^{\frac{N}{2}-1}-1}{2^{\frac{N}{2}-1}} \delta=\alpha$,

which contradicts the fact that $\alpha_{t_{0}+k-1} \geq \alpha$, which implies $\min _{j \in E_{t}} x_{j}^{t} \stackrel{t \rightarrow \infty}{\longrightarrow} \alpha$. In the same way, we can prove that $\min _{j \in O_{t}} x_{j}^{t} \stackrel{t \rightarrow \infty}{\longrightarrow} \beta$. It then holds that $\lim _{t \rightarrow \infty} F^{2 t}(\boldsymbol{x})=(\alpha \beta)^{\frac{N}{2}}$ and $\lim _{t \rightarrow \infty} F^{2 t+1}(\boldsymbol{x})=(\beta \alpha)^{\frac{N}{2}}$. 
According to Eqs. 111 and (13), the numbers $\alpha$ and $\beta$ satisfy the following equations:

$$
\left\{\begin{array}{l}
\alpha=\alpha(1-(1-2 d) \beta) \\
\beta=\beta(1-(1-2 d) \alpha) \\
\frac{N}{2}(\alpha-\beta)=x_{0}-x_{1}+x_{2}-x_{3}+\ldots-x_{N-1} .
\end{array}\right.
$$

The first two imply that if $\alpha \neq 0$, then $\beta=0$ and vice versa. Therefore, if $x_{0}-x_{1}+x_{2}-x_{3}+\ldots-x_{N-1} \geq 0$, then $\alpha=\frac{2}{N}\left(x_{0}-x_{1}+x_{2}-x_{3}+\ldots-x_{N-1}\right)$ and $\beta=0$. Otherwise, $\alpha=0$ and $\beta=-\frac{2}{N}\left(x_{0}-x_{1}+x_{2}-x_{3}+\ldots-\right.$ $\left.x_{N-1}\right)$. This concludes the proof for even $N$.

Second, we consider odd $N$. Consider the following concatenation:

$(\boldsymbol{x} \boldsymbol{x})=x_{0} x_{1} \ldots x_{N-1} x_{0} x_{1} \ldots x_{N-1} \in X_{2 N}$.

Due to the periodic boundary conditions, it holds for each $t \geq 0$ that

$F^{t}(\boldsymbol{x} \boldsymbol{x})=F^{t}(\boldsymbol{x}) F^{t}(\boldsymbol{x})$.

However, for the initial configuration $\boldsymbol{x} \boldsymbol{x}$, the alternating sum equals 0 and the first part of the theorem implies that

$\lim _{t \rightarrow \infty} F^{2 t}(\boldsymbol{x} \boldsymbol{x})=\lim _{t \rightarrow \infty} F^{2 t+1}(\boldsymbol{x} \boldsymbol{x})=0^{2 N}$.

This concludes the proof for odd $N$.

4.4.5 The case $(b, c, d)=(0,1, d)$ for $\frac{1}{2} \leq d<1$.

In this subsection, we discuss the dynamics of local rules parameterized by points belonging to the edge $P_{5} P_{7}$. In this case, $\lambda=0$ and the local rule is given by

$f(x, y, z)=y(1-(1-d)(x+z))$.

In particular, $f(x, 0, z)=0$ and $f(0, y, 0)=y$. This means that any binary configuration $\boldsymbol{x} \in X_{N}$ not containing two neighboring $1 \mathrm{~s}$ is a fixed point of $f$. Furthermore, each substring of $\boldsymbol{x} \in X_{N}$ starting and ending with 0 and not containing two neighboring 1 s remains unchanged in $F^{t}(\boldsymbol{x})$ for every $t \geq 0$. Additionally, according to Lemma 4 we have $F^{t}\left(1^{N}\right) \stackrel{t \rightarrow \infty}{\longrightarrow} 0^{N}$.

For these reasons, to uncover the behavior of $f$, it suffices to consider strings of the type $011 \ldots 110$, where the number of 1 s equals $K$ with $2 \leq K<N$. W.l.o.g., let us assume that $x_{0}^{0}=0, x_{1}^{0}=x_{2}^{0}=\ldots=x_{K}^{0}=$ $1, x_{K+1}^{0}=0$. The dynamics of $f$ on $x_{0}^{0} x_{1}^{0} \ldots x_{K}^{0} x_{K+1}^{0}=$ $011 \ldots 110$ is then described by the system of recurrence relations:

$$
\left\{\begin{array}{l}
x_{0}^{t+1}=x_{K+1}^{t+1}=0 \\
x_{n}^{t+1}=x_{n}^{t}\left(1-(1-d)\left(x_{n-1}^{t}+x_{n+1}^{t}\right)\right), n \in\{1, \ldots, K\},
\end{array}\right.
$$

where $\frac{1}{2} \leq d<1$. Since $0 \leq 1-(1-d)\left(x_{n-1}^{t}+x_{n+1}^{t}\right) \leq$ 1 , the sequence $\left(x_{n}^{t}\right)_{t=0}^{\infty}$ is decreasing for every $n \in$ $\{1,2, \ldots, K\}$ and thus converges. We denote this limit as $q_{n}=\lim _{t \rightarrow \infty} x_{n}^{t} \in[0,1]$. According to Eq. [15), it holds that

$q_{n}=q_{n}\left(1-(1-d)\left(q_{n-1}+q_{n+1}\right)\right)$,

for any $n \in\{1,2, \ldots, K\}$, where $q_{0}=q_{K+1}=0$. Since $f$ is outer-totalistic, it holds that

$x_{n}^{t}=x_{K+1-n}^{t}$

for any $n \in\{1,2, \ldots, K\}$ and any $t \geq 0$, and thus

$q_{n}=q_{K+1-n}$

for any $n \in\{1,2, \ldots, K\}$.

We start with some technical lemmata.

Lemma 7 For each $n \in\{1,2, \ldots, K-1\}$, it holds that $q_{n} q_{n+1}=0$. Moreover, if $K$ is even, then $q_{\frac{K}{2}}=q_{\frac{K}{2}+1}=$ 0 . In particular, if $K=2$, then $q_{1}=q_{2}=0$.

Proof Since, $\frac{1}{2} \leq d<1$, it follows from Eq. (16) that $q_{n-1} q_{n}+q_{n} q_{n+1}=0$, for any $n \in\{1,2, \ldots, K-1\}$. Since $q_{0}=0$, we get $q_{1} q_{2}=0$. A simple induction yields the first part of the claim. If $K$ is even, then, according to Eq. (18), it holds for $n=\frac{K}{2}$ that $q_{\frac{K}{2}}=q_{\frac{K}{2}+1}$. Hence, $q_{\frac{K}{2}} q_{\frac{K}{2}+1}=0$ implies $q_{\frac{K}{2}}=q_{\frac{K}{2}+1}=0$, which concludes the proof.

The above lemma states that regardless of $d \in\left[\frac{1}{2}, 1\right]$, the string 0110 goes to 0000 :

$\ldots 0110 \ldots \stackrel{t \rightarrow \infty}{\longrightarrow} \ldots 0000 \ldots$.

From here on, we can assume that $K \geq 3$.

Lemma 8 If $K \geq 3$, then $q_{1} \geq 1-d$. Moreover, if $d=$ $\frac{1}{2}$, then the string $011 \ldots 110$ in $\boldsymbol{x}$ turns into the string $0 \frac{1}{2} 0 \ldots 0 \frac{1}{2} 0$ in $F(\boldsymbol{x})$, which then remains unchanged in $F^{t}(\boldsymbol{x})$ for any $t \geq 1$.

Proof According to Eq. 15, for any $t>0$, it holds that

$x_{1}^{t}-x_{2}^{t}=x_{1}^{t-1}-x_{2}^{t-1}+(1-d) x_{2}^{t-1} x_{3}^{t-1}$.

Hence, also using $x_{1}^{0}=x_{2}^{0}=1$, we have

$x_{1}^{t}-x_{2}^{t}=x_{1}^{0}-x_{2}^{0}+(1-d) \sum_{l=0}^{t-1} x_{2}^{l} x_{3}^{l}=(1-d) \sum_{l=0}^{t-1} x_{2}^{l} x_{3}^{l}$.

Since $x_{2}^{0} x_{3}^{0}=1$ and $x_{2}^{l} x_{3}^{l} \geq 0$, for any $l \geq 0$, it then follows that

$q_{1} \geq q_{1}-q_{2}=(1-d) \sum_{l=0}^{\infty} x_{2}^{l} x_{3}^{l} \geq 1-d$.

Further, if $d=\frac{1}{2}$, then the local rule is given by $f(x, y, z)=y\left(1-\frac{1}{2}(x+z)\right)$, and the second part of the lemma readily follows. 
Finally, we consider the case $\frac{1}{2}<d<1$ and $K \geq 3$. The following theorem describes the dynamics of $F$ on strings $011 \ldots 110$, where the number of 1 s is even.

Theorem 8 Let $\frac{1}{2}<d<1$ and $K \geq 4$ be even. For any $t \geq 0$, it holds that

$x_{1}^{t} \geq x_{3}^{t} \geq \ldots \geq x_{K-3}^{t} \geq x_{K-1}^{t}$

and, as a consequence,

$q_{1} \geq q_{3} \geq \ldots \geq q_{K-3} \geq q_{K-1}$.

Moreover, $q_{2 j}=q_{K+1-2 j}=0$, for $2 j \leq \frac{K}{2}$.

Proof To prove Eq. (19), we apply induction w.r.t. $t$. For $t=0$, these inequalities are obvious. Assume that they are satisfied for some $t \geq 0$. Using the symmetry relationships in Eq. (17), we have

$x_{2 j}^{t} \leq x_{2 j+2}^{t}$

for any $j \in\left\{1,2, \ldots, \frac{K}{2}-1\right\}$. Using the induction hypothesis, it then follows that

$$
\begin{aligned}
x_{2 j-1}^{t+1} & =x_{2 j-1}^{t}\left(1-(1-d)\left(x_{2 j-2}^{t}+x_{2 j}^{t}\right)\right) \\
& \geq x_{2 j+1}^{t}\left(1-(1-d)\left(x_{2 j}^{t}+x_{2 j+2}^{t}\right)\right)=x_{2 j+1}^{t+1},
\end{aligned}
$$

for any $j \in\left\{1,2, \ldots, \frac{K}{2}-1\right\}$, which yields Eq. 119 and thus Eq. 201. According to Lemma 7, it holds that $q_{\frac{K}{2}}=q_{\frac{K}{2}+1}=0$, so Eq. (20) implies that $q_{2 j+1}=0$, for any $2 j+1 \geq \frac{K}{2}$. Thus

$q_{2 j}=q_{K+1-2 j}=0$,

for any $2 j \leq \frac{K}{2}$, which concludes the proof.

The above theorem can be illustrated as follows. Suppose that in a string $011 \ldots 110$ the number of $1 \mathrm{~s}$ is even, then

$$
\begin{aligned}
& \begin{array}{lllllllllllll}
0 & 1 & 1 & 1 & \ldots & 1 & \vdots & 1 & \ldots & 1 & 1 & 1 & 0
\end{array} \\
& \downarrow t \rightarrow \infty \\
& \begin{array}{lllllllllllllllll}
0 & q_{1} & 0 & q_{3} & \ldots & 0 & \vdots & 0 & \ldots & q_{3} & 0 & q_{1} & 0
\end{array}
\end{aligned}
$$

where $q_{1} \geq q_{3} \geq \ldots$ and $q_{1} \geq 1-d$.

For an odd number $K \geq 3$, we introduce the following notations: $s=\frac{K+1}{2}, 2 n_{o}-1$ is the largest odd number not greater than $s$ and $2 n_{e}$ is the largest even number not greater than $s$.

Lemma 9 Let $K \geq 3$ be odd. For any $t \geq 0$, it holds that

$x_{1}^{t} \geq x_{3}^{t} \geq \ldots \geq x_{2 n_{o}-1}^{t}$

$x_{2}^{t} \leq x_{4}^{t} \leq \ldots \leq x_{2 n_{e}}^{t}$.
Additionally,

$x_{2 n_{o}-1}^{t} \geq x_{2 n_{e}}^{t}$.

Moreover, if $t>s$, then the inequalities in Eqs. (21) and (22) are strict.

Proof One can prove Eq. 21) using induction in a similar way as in the proof of Theorem 8. We can also prove Eq. (22) using induction. Note that for $t=0$ the inequality is trivially fulfilled and assume that it is satisfied for some $t \geq 0$. If $s$ is odd, then $2 n_{o}-1=s$ and $2 n_{e}=s-1$, thus Eq. 21) and the induction hypothesis imply

$$
\begin{aligned}
x_{2 n_{e}}^{t+1}=x_{s-1}^{t+1} & =x_{s-1}^{t}\left(1-(1-d)\left(x_{s-2}^{t}+x_{s}^{t}\right)\right) \\
& \leq x_{s}^{t}\left(1-(1-d)\left(x_{s}^{t}+x_{s}^{t}\right)\right) \\
& \leq x_{s}^{t}\left(1-(1-d)\left(x_{s-1}^{t}+x_{s-1}^{t}\right)\right) \\
& =x_{s}^{t}\left(1-(1-d)\left(x_{s-1}^{t}+x_{s+1}^{t}\right)\right) \\
& =x_{s}^{t+1}=x_{2 n_{o}-1}^{t+1} .
\end{aligned}
$$

Similarly, if $s$ is even, then $2 n_{e}=s$ and $2 n_{o}-1=s-1$, and it follows using Eq. (21), the induction hypothesis and the fact that $x_{s-1}^{t}=x_{s+1}^{t}$ according to Eq. (17), that

$$
\begin{aligned}
x_{2 n_{e}}^{t+1}=x_{s}^{t+1} & =x_{s}^{t}\left(1-(1-d)\left(x_{s-1}^{t}+x_{s+1}^{t}\right)\right) \\
& \leq x_{s}^{t}\left(1-(1-d)\left(x_{s}^{t}+x_{s}^{t}\right)\right) \\
& \leq x_{s-1}^{t}\left(1-(1-d)\left(x_{s-2}^{t}+x_{s}^{t}\right)\right)=x_{s-1}^{t+1} \\
& =x_{2 n_{o}-1}^{t+1} .
\end{aligned}
$$

Observe that if $2 j+1<\frac{K}{2}$ and $x_{2 j-1}^{t}>x_{2 j+1}^{t}$, or $x_{2 j-2}^{t}<x_{2 j}^{t}$, or $x_{2 j}^{t}<x_{2 j+2}^{t}$, then $x_{2 j-1}^{t+1}>x_{2 j+1}^{t+1}$ and, consequently, $x_{2 j-1}^{t+k}>x_{2 j+1}^{t+k}$ for $k \geq 1$. Analogously, if $2 j+2<\frac{K}{2}$ and $x_{2 j}^{t}<x_{2 j+2}^{t}$, or $x_{2 j-1}^{t}>x_{2 j+1}^{t}$, or $x_{2 j+1}^{t}>x_{2 j+3}^{t}$, then $x_{2 j}^{t+1}<x_{2 j+2}^{t+1}$ and, consequently, $x_{2 j}^{t+k}<x_{2 j+2}^{t+k}$ for $k \geq 1$. Since $x_{1}^{1}=1-(1-d)>$ $1-2(1-d)=x_{3}^{1}$, by simple induction one can obtain strict inequalities in Eq. (21) for $t \geq s$. This also implies that the inequalities in Eq. (22) are strict.

Lemma 10 Let $K \geq 3$ be odd. For any $t \geq 0$, it holds that

$x_{2 n_{o}-1}^{t+1}-x_{2 n_{e}}^{t+1} \geq x_{2 n_{o}-1}^{t}-x_{2 n_{e}}^{t}$.

Proof For odd $s$, we have $x_{2 n_{e}}^{t}=x_{2 n_{e}+2}^{t}$, thus

$$
\begin{aligned}
x_{2 n_{o}-1}^{t+1}-x_{2 n_{e}}^{t+1}= & x_{2 n_{o}-1}^{t}\left(1-(1-d)\left(x_{2 n_{e}}^{t}+x_{2 n_{e}}^{t}\right)\right) \\
& -x_{2 n_{e}}^{t}\left(1-(1-d)\left(x_{s-2}^{t}+x_{2 n_{o}-1}^{t}\right)\right) \\
= & x_{2 n_{o}-1}^{t}-x_{2 n_{e}}^{t} \\
& -(1-d) x_{2 n_{e}}^{t}\left(x_{2 n_{o}-1}^{t}-x_{s-2}^{t}\right) \\
\geq & x_{2 n_{o}-1}^{t}-x_{2 n_{e}}^{t} .
\end{aligned}
$$


For even $s$, we have $x_{2 n_{o}-1}^{t}=x_{2 n_{o}+1}^{t}$, thus

$$
\begin{aligned}
x_{2 n_{o}-1}^{t+1}-x_{2 n_{e}}^{t+1}= & x_{2 n_{o}-1}^{t}-x_{2 n_{e}}^{t} \\
& -(1-d) x_{2 n_{o}-1}^{t}\left(x_{s-2}^{t}-x_{2 n_{e}}^{t}\right) \\
\geq & x_{2 n_{o}-1}^{t}-x_{2 n_{e}}^{t} .
\end{aligned}
$$

Theorem 9 Let $K \geq 3$ be odd. For any $2 j-1 \leq s$, it holds that $q_{2 j-1}>0$, while for any $2 j \leq s$, it holds that $q_{2 j}=0$. Moreover, $q_{1}+q_{3}+\ldots+q_{K}=1$.

Proof If $t>s$, then for $2 j-1 \leq s$ Lemmata 9 and 10 imply that

$x_{2 j-1}^{t} \geq x_{2 n_{o}-1}^{t} \geq x_{2 n_{o}-1}^{t}-x_{2 n_{e}}^{t} \geq x_{2 n_{o}-1}^{s+1}-x_{2 n_{e}}^{s+1}$.

Thus $q_{2 j-1} \geq x_{2 n_{o}-1}^{s+1}-x_{2 n_{e}}^{s+1}>0$, since for $t=s+1$ inequality Eq. 22 is strict. Furthermore, since $q_{2 j-1}>$ 0 , Lemma 7 implies that $q_{2 j}=0$. To prove the last part of the theorem, note that since $x_{0}^{t}=x_{K+1}^{t}=0$, it holds that

$$
\begin{aligned}
\sum_{j=1}^{K}(-1)^{j+1} x_{j}^{t} & =\sum_{j=1}^{K}(-1)^{j+1} x_{j}^{t-1} \\
& =\ldots=\sum_{j=1}^{K}(-1)^{j+1} x_{j}^{0}=1 .
\end{aligned}
$$

This implies that $\sum_{j=1}^{K}(-1)^{j+1} q_{j}=1$. Since $q_{j}=0$ for even $j$, the proof is complete.

The above theorem can be illustrated as follows. For even $s$, we have

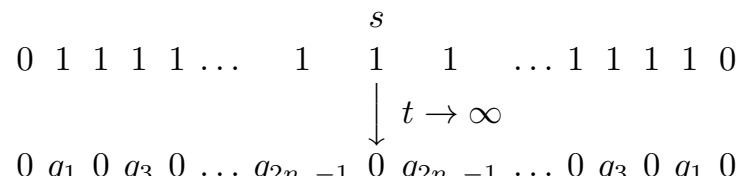

while for odd $s$, we have

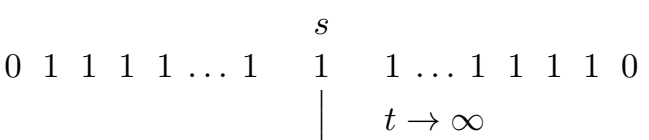

$$
\begin{aligned}
& \begin{array}{lllllllllllllllllllll}
0 & q_{1} & 0 & q_{3} & 0 & \ldots & 0 & q_{2 n_{o}-1} & 0 & \ldots & 0 & q_{3} & 0 & q_{1} & 0
\end{array}
\end{aligned}
$$

\section{Conclusions}

In this paper, we have provided a complete description of the dynamics of legal outer-totalistic ACCAs. On the one hand, such CAs are the simplest generalization of Elementary Cellular Automata, while on the other hand, they are dynamical systems that exhibit some properties that do not occur in the binary case. Thanks to massive numerical simulations, we have been able to partition the rule space in a number of classes with a distinct behavior. Through the use of a panoply of proof techniques and oftentimes tedious proofs, we have been able to provide undeniable analytical evidence for the dynamics observed.

Table 4 summarizes the results obtained. In order to keep the presentation manageable, we focus on initial configurations from $X_{N}^{0,1}$ only and add a short explanation for the other configurations separately below the table.

Since we only consider legal outer-totalistic ACCAs, it always holds that $F^{t}\left(0^{N}\right)=0^{N}$. For the configuration $x=1^{N}$, the dynamics is as follows: if $2 d-c=0$ or $2 d-$ $c=1$, then $F^{t}\left(1^{N}\right)=0^{N}$ or $F^{t}\left(1^{N}\right)=1^{N}$, respectively, while if $0<2 d-c<1$, then $F^{t}\left(1^{N}\right)$ tends to the homogeneous configuration $0^{N}$ (if $2 b+c \leq 1$ ) or $\lambda^{N}$ (if $2 b+c>1)$.

The results obtained show that in the set of ACCAs considered, one can observe various types of sensitivity:

- Sensitivity to the change of a single value in an initial configuration. This kind of sensitivity may concern all initial configurations, as for example, for $f(x, y, z)=0.4(x+z)+0.2 y(\operatorname{see}(\mathrm{s} 3)$ in Theorem 3$)$ or only some initial configurations, as for example, for $f(x, y, z)=0.2(x+z) y+0.4(x+z)-0.2 y($ see $(\mathrm{s} 1)$ in Theorem 3.

- Sensitivity to the change of the number of cells in the grid. Note that if we add one cell to the considered grid, then the parity of $N$ will change. For example, the local rule $f(x, y, z)=-0.1(x+z) y+0.5(x+$ $z)$ is not immune to such interference in the grid structure (see Theorem 7).

- Sensitivity to slight changes in the parameters of a local rule. Perhaps the best example of this sensitivity are local rules parameterized by points lying on the edge $P_{1} P_{3}$, i.e., local rules given by the following expression:

$$
f(x, y, z)=-\left(\frac{1}{2}-d\right)(x+z) y+\frac{1}{2}(x+z)
$$

where $0 \leq d \leq \frac{1}{2}$. The dynamics of such ACCAs for $0 \leq d<\frac{1}{2}$ (see Theorem 7) is completely different than for $d=\frac{1}{2}$ (see (s3) in Theorem 3).

Although we limited our investigation to binary initial configuration only, nearly all results can be readily extended to all configurations from $[0,1]^{N}$. However, the case described in Subsection 4.4.5 would require a more substantial effort.

Acknowledgements The computations were carried out at the Academic Computer Centre in Gdańsk (TASK KDM). 
Table 4: Classes of ACCA rules with distinct dynamical behavior.

\begin{tabular}{|c|c|c|c|c|}
\hline \multicolumn{4}{|c|}{ Conditions } & Dynamics for $\boldsymbol{x} \in X_{N}^{0,1}$ \\
\hline \multirow{6}{*}{$\begin{array}{l}\overrightarrow{\|} \\
\| \\
0 \\
\dot{J} \\
\widetilde{N}\end{array}$} & \multicolumn{3}{|c|}{$b+d<1$} & $F^{t}(\boldsymbol{x}) \rightarrow 0^{N}$ \\
\hline & \multicolumn{3}{|c|}{$b+d>1$} & $F^{t}(\boldsymbol{x}) \rightarrow 1^{N}$ \\
\hline & \multirow{4}{*}{$b+d=1$} & \multicolumn{2}{|c|}{$0<b<\frac{1}{2}$} & $F^{t}(\boldsymbol{x}) \rightarrow(\rho(\boldsymbol{x}))^{N}$ \\
\hline & & \multicolumn{2}{|c|}{$b=0$} & $F^{t}(\boldsymbol{x})=\boldsymbol{x}$ \\
\hline & & \multirow[b]{2}{*}{$b=\frac{1}{2}$} & $N$ odd & $F^{t}(\boldsymbol{x}) \rightarrow(\rho(\boldsymbol{x}))^{N}$ \\
\hline & & & $N$ even & $\begin{array}{l}F^{2 t}(\boldsymbol{x}) \rightarrow\left(\rho_{0}(\boldsymbol{x}) \rho_{1}(\boldsymbol{x})\right)^{\frac{N}{2}} \\
F^{2 t+1}(\boldsymbol{x}) \rightarrow\left(\rho_{1}(\boldsymbol{x}) \rho_{0}(\boldsymbol{x})\right)^{\frac{N}{2}}\end{array}$ \\
\hline \multirow{8}{*}{$\begin{array}{l}\vec{v} \\
v \\
0 \\
1 \\
\vec{\sim} \\
\text { VI } \\
0\end{array}$} & \multicolumn{3}{|c|}{$2 b+c<1$} & $F^{t}(\boldsymbol{x}) \rightarrow 0^{N}$ \\
\hline & $2 b+c \geq 1$ & \multicolumn{2}{|c|}{$0<b<\frac{1}{2} \vee 0<c<1$} & $F^{t}(\boldsymbol{x}) \rightarrow \lambda^{N}$ \\
\hline & \multirow{3}{*}{$b=\frac{1}{2} \wedge c=1$} & \multirow[b]{2}{*}{$d=\frac{1}{2}$} & $N$ odd & $F^{t}(\boldsymbol{x}) \rightarrow \lambda^{N}$ \\
\hline & & & $N$ even & $\begin{array}{l}F^{t}(\boldsymbol{x}) \rightarrow \lambda^{N} \text { if } \boldsymbol{x} \notin\left\{(01)^{\frac{N}{2}},(10)^{\frac{N}{2}}\right\} \\
F^{t}(\boldsymbol{x}) \rightarrow 0^{N} \text { if } \boldsymbol{x} \in\left\{(01)^{\frac{N}{2}},(10)^{\frac{N}{2}}\right\}\end{array}$ \\
\hline & & & & $F^{t}(\boldsymbol{x}) \rightarrow \lambda^{N}$ \\
\hline & \multirow[b]{2}{*}{$b=\frac{1}{2} \wedge c=0$} & \multicolumn{2}{|c|}{$N$ odd } & $F^{t}(\boldsymbol{x}) \rightarrow 0^{N}$ \\
\hline & & \multicolumn{2}{|c|}{$N$ even } & $\begin{aligned} F^{2 t+\varepsilon}(\boldsymbol{x}) & \rightarrow(|\alpha| 0)^{\frac{N}{2}} \\
F^{2 t+1+\varepsilon}(\boldsymbol{x}) & \rightarrow(0|\alpha|)^{\frac{N}{2}}\end{aligned}, \varepsilon=\left\{\begin{aligned} 0 & , \alpha \geq 0 \\
1 & , \alpha<0\end{aligned}\right.$ \\
\hline & \multicolumn{3}{|c|}{$b=0 \wedge c=1$} & $\begin{array}{l}\text { Each segment } 01^{k} 0 \text { has its own dynamics } \\
\text { (see Subsection } 4.4 .5\end{array}$ \\
\hline
\end{tabular}

\section{Data Availability}

Data sharing is not applicable to this article as any datasets were generated by presented formulas and analysed during the current study.

\section{Conflict of interest}

The authors declare that they have no conflict of interest.

\section{References}

1. Kaneko, K.: Coupled map lattice. In: Artuso, R., Cvitanović, P., Casati, G. (eds.) Chaos, Order, and Patterns. NATO ASI Series, vol. 280, pp. 237-247. Springer, Boston, MA (1991). https://doi.org/10.1007/ 978-1-4757-0172-2_1

2. Kaneko, K.: Overview of coupled map lattices. Chaos: An Interdisciplinary Journal of Nonlinear Science 2(3), 279-282 (1992)

3. Liu, Z., Wang, Y., Zhao, Y., Zhang, L.Y.: A stream cipher algorithm based on 2D coupled map lattice and partitioned cellular automata. Nonlinear Dynamics 101, 1383-1396 (2020)

4. Cattaneo, G., Flocchini, P., Mauri, G., Vogliotti, C.Q., Santoro, N.: Cellular automata in fuzzy backgrounds. Physica D: Nonlinear Phenomena 105(1), 105-120 (1997)
5. Gang, H., Zhilin, Q.: Controlling spatiotemporal chaos in coupled map lattice systems. Phys. Rev. Lett. 72, 68-71 (1994)

6. Cánovas, J.S., Linero Bas, A., Soler López, G.: Chaotic synchronization in a type of coupled lattice maps. Communications in Nonlinear Science and Numerical Simulation 62, 418-428 (2018)

7. Ibrahimi, M., Gulseren, O., Jahangirov, S.: Deterministic phase transitions and self-organization in logistic cellular automata. Physical Review E 100(4), 042216 (2019)

8. Betel, H., Flocchini, P.: On the asymptotic behavior of fuzzy cellular automata. Electronic Notes in Theoretical Computer Science 252, 23-40 (2009)

9. Wolnik, B., Dembowski, M., Bołt, W., Baetens, J.M., De Baets, B.: Density-conserving affine continuous cellular automata solving the relaxed density classification problem. Journal of Physics A: Mathematical and Theoretical 50(34), 345103 (2017)

10. Briceño, R., de Espanés, P.M., Osses, A., Rapaport, I.: Solving the density classification problem with a large diffusion and small amplification cellular automaton. Physica D: Nonlinear Phenomena 261, 70-80 (2013)

11. Flocchini, P., Geurts, F., Mingarelli, A., Santoro, N.: Convergence and aperiodicity in fuzzy cellular automata: Revisiting rule 90. Physica D: Nonlinear Phenomena 142(1), 20-28 (2000)

12. Betel, H., Flocchini, P.: On the relationship between Boolean and fuzzy cellular automata. Electronic Notes in Theoretical Computer Science 252, 5-21 (2009)

13. Bołt, W., Baetens, J.M., De Baets, B.: On the decomposition of stochastic cellular automata. Journal of Computational Science 11, 245-257 (2015). https://doi.org/ $10.1016 / \mathrm{j} \cdot$ jocs.2015.09.004 
14. Gardner, M.: Mathematical games - the fantastic combinations of john conway's new solitaire game "life". In: Scientific American vol. 223, pp. 120-123. Scientific American, a division of Nature America, Inc., (1970)

15. Eppstein, D.: Growth and decay in life-like cellular automata. In: Adamatzky, A. (ed.) Game of Life Cellular Automata, pp. 71-97. Springer, London (2010)

16. Kawano, T.: Translating the conway's game of life as a discrete logistic cellular automata model with density effects. International Journal of Innovative Computing, Information and Control 16(5), 1655-1666 (2020)

17. Turney, P.D.: Measuring Behavioral Similarity of Cellular Automata. Artificial Life 27(1), 62-71 (2021). https:// doi.org/10.1162/artl_a_00337

18. Vieira, A.P., Goles, E., Herrmann, H.J.: Phase transitions in a conservative game of life. Phys. Rev. E 103, 012132 (2021). https://doi.org/10.1103/PhysRevE.103.012132

19. Wolfram, S.: Statistical mechanics of cellular automata. Rev. Mod. Phys. 55, 601-644 (1983)

20. Roth, T.O.: About the robustness of $1 \mathrm{~d}$ cellular automata revising their temporal entropy. Physica D: Nonlinear Phenomena 425, 132953 (2021). https://doi.org/ $10.1016 / j$.physd.2021.132953

21. El Yacoubi, S., Mingarelli, A.: An algebraic characterization of fuzzy cellular automata. Journal of Cellular Automata 6, 195-206 (2011)

22. Flocchini, P., Cezar, V.: Radial view of continuous cellular automata. Fundamenta Informaticae 87(2), 165-183 (2008) 